\title{
Análisis de la producción científica en el uso de la realidad virtual en la educación a partir de la estructura conceptual, social e intelectual.
}

\section{Analysis of the scientific production in the use of virtual reality in education using the conceptual, social and intellectual structure.}

\author{
Gonzalo Lorenzo Lledó \\ Universidad de Alicante, Alicante, España. \\ glledo@ua.es
}

\begin{abstract}
Resumen
En los últimos años el uso de la tecnología en la educación ha dado lugar a un aumento de la producción científica. Entre las diversas tecnologías, la realidad virtual está adquiriendo un mayor uso en las aulas educativas. Sin embargo, aún no existe una imagen global de cómo está cambiando la producción de investigaciones sobre el uso de la realidad virtual en la educación. En este sentido, el objetivo de este estudio es analizar la evolución en la producción científica sobre el uso de la realidad virtual en la educación mediante la aproximación bibliométrica del mapeado científico a partir del estudio de la estructura conceptual, social e intelectual del campo de conocimiento. La muestra está compuesta de 1163 documentos obtenidos de la Web of Science durante el periodo 1996-2019. Los resultados muestran que existen dos líneas de investigación: una de ellas Tecnología y Educación mientras que la otra es Medicina y Educación. Mientras que las redes de colaboración más importantes la forman los autores más productivos. Asimismo, los documentos de autores como Merchant et al., (2014) y Nicholson et al., (2006) son elementos de referencia para el trabajo de la realidad virtual en educación.

Palabras clave: Educación, aprendizaje, Tecnología educativa, Bibliometría, redes, producción científica.
\end{abstract}

\begin{abstract}
In recent years, the use of technology in education has led to an increase in scientific production. Among the various technologies, virtual reality is gaining greater use in educational classrooms. However, there is still no global picture of how research production on the use of virtual reality in education is changing. In this sense, the aim of this study is to analyze the evolution of scientific production on the use of virtual reality in education through the bibliometric approach of scientific mapping based on the study of the conceptual, social, and intellectual structure of the field of knowledge. The sample is composed of 1163 documents obtained from the Web of Science during the period 1996-2019. The results show that there are two lines of research: one of them Technology and Education while the other is Medicine and Education. While the most important collaborative networks are formed by the most productive authors. Likewise, the papers of authors such as Merchant et al., (2014) and Nicholson et al., (2006) are key elements for the work of virtual reality in education.
\end{abstract}

Key words: Education, Learning, Educational Technology, Bibliometrics, networks, scientific production. 


\section{1. - Introducción.}

En las sociedades actuales la tecnología tiene un papel fundamental, cada vez son más las actividades de la vida diaria que se llevan a cabo con estas herramientas llamadas Tecnologías de la Información y la Comunicación (TIC) Estas ofrecen soluciones a problemas de distinta índole mediante la creación e intercambio de diversidad de contenidos electrónicos (Pérez-Mercado et al., 2018). Según Fernández-Cruz y Fernández-Díaz, (2018) uno de los campos donde mayor difusión ha tenido las TIC es la educación. La implantación de esta nueva herramienta ha dado lugar a nuevos métodos de enseñanza (Viñals y Cuenca, 2016). Asimismo, ha producido una mejora en la motivación, adaptación a las necesidades del alumnado y participación en los procesos de aprendizaje (Marín y Muñoz, 2018). Sin embargo, las TIC pueden generar distracciones en los estudiantes durante el desarrollo de las actividades.

Dentro del conjunto de prácticas innovadoras que se han puesto en marcha en los centros educativos cabe destacar el trabajo mediante la realidad virtual (RV). En este sentido, las tecnologías de realidad virtual como indican Newbutt et al., (2016) se dividen en varios tipos la RV basada en Head Mounted Display (HMD), simulaciones de entornos de realidad virtual, entornos virtuales colaborativos, entornos virtuales inmersivos y mundo virtuales. La diferencia principal dentro de cada uno de los tipos es la inmersividad a la que está sometida el usuario y los diferentes dispositivos de comunicación de entrada y salida que se utilizan. Asimismo, uno de los tipos de realidad virtual que está teniendo un mayor uso en los entornos educativos debido a su bajo coste es la realidad virtual basada en video esférico (Schmidt, et al. 2021). Según estos autores, este tipo utiliza videos de 360 grados para renderizar la representación del entorno virtual para ello utiliza los dispositivos HMD mediante entornos inmersivos. Además, el usuario puede interactuar con el contenido y el contexto a través de los movimientos de su cabeza (Hwang et al., 2018). En otra clasificación más general de la RV, Hamilton et al., (2021) describen dos grandes grupos: la RV de escritorio y la RV inmersiva. La RV de escritorio el usuario manipula el entorno virtual en la pantalla de un ordenador, mientras que la RV inmersiva utiliza dispositivos HMD y visuales de $360^{\circ}$ mediante videos. A partir de los diversos tipos de realidad virtual se va a realizar una caracterización de esta herramienta según diversas investigaciones (Biocca, 1992; Burdea y Coiffet, 1994; Johnson et al., 1998; Lee y Wang, 2014; Parsons y Cobb, 2011). Estos autores indica que la RV es un entorno creado por un ordenador donde el usuario se siente presente en dicha actividad, además la RV puede ser diseñada como un interfaz de usuario de alta gama. Esto implica la simulación real y la interacción a través de múltiples canales sensoriales. Otro aspecto importante resaltado por estas investigaciones es la variedad de dispositivos de interacción que se utilizan y la gran resolución en 3D de los entornos diseñados.

Por otro lado, el uso de la RV está cada vez más difundida en la pedagogía del aprendizaje (Sural, 2018) y su formación se está incorporando cada vez más en los planes de estudio debido a que es una modalidad eficaz de evaluación y formación (Abi-Rafeh et al., 2019; Huttar y Brintzenhofeszoc, 2019). De igual manera, la realidad virtual presenta una serie de bondades dentro del campo educativo como por ejemplo la mejor comprensión de los contenidos, un incremento en el rendimiento de los estudiantes, una mejora en la motivación y la creatividad, una intensificación de la motivación debido a que aísla los sentidos del usuario, además de una mayor participación en las dinámicas educativas

Análisis de la producción científica en el uso de la realidad virtual en la educación a partir de la estructura conceptual, social e intelectual. Gonzalo Lorenzo Lledó

Página $\mathbf{2}$ de $\mathbf{3 1}$ 
(Hanson et al., 2019; Kim y Hall, 2019; Marín y Muñoz, 2018). Además, hace la educación accesible aumentando las competencias de los discentes (Wu et al., 2019). Otros autores como Chávez y Bayona (2018) descubrieron que, en ciertas materias como la medicina, la característica de "movimiento" es vital para aprender sobre la reacción del cuerpo de una persona, mientras que, en la educación general, las "interfaces de inmersión" se utilizan a menudo como una forma de aprender a través de una "experiencia en vivo" más cercana a la realidad. Además para estos autores la RV tiene diferentes efectos positivos en el aprendizaje como son el aumento del interés, la mejora de las competencias, la vivencia a experiencias cercanas a la realidad. En esta línea Bower et al. (2020) aseguran que la realidad virtual inmersiva (RVI) tiene el potencial de aumentar la inmersión, mejorar las capacidades espaciales, promover la empatía, aumentar la motivación y mejorar los resultados del aprendizaje. Asimismo desde el punto de vista del aprendizaje la RV según Liu et al. (2017) proporciona una serie de contextos, actividades, e interacciones sociales dentro de los entornos de aprendizaje que hacen posible que el estudiante sea el centro del aprendizaje, además de un elemento activo de procesamiento de la información (Cunningham y Duffy, 1996).

\section{1.- Revisión conceptual de la bibliometría.}

En los últimos años son cada vez más las investigaciones que utiliza la bibliometría para llevar a cabo una revisión de la literatura científica (Aria et al., 2020). En este sentido, para desarrollar un estudio sobre un campo de conocimiento Noyons et al., (1999) afirman que existen dos tipos de aproximaciones bibliométricas: el análisis de rendimiento que utiliza unos indicadores como son los años de publicación, la cantidad de documentos publicados anualmente o las áreas de conocimiento. El segundo tipo según Noyons et al., (1999) es el mapeado científico. Este enfoque según Martínez et al., (2015) tiene como objetivo el descubrimiento de la estructura conceptual de la producción científica mediante mapas de ciencia. De manera más concreta, Martínez et al., (2015) concluyen que el mapeado científico se centra en el seguimiento de un campo científico y en la delimitación de áreas de investigación para determinar su estructura conceptual y su evolución científica. Los mapas científicos según Callon et al., (1983) pueden construirse mediante el análisis de co-palabras. Esta técnica utiliza las palabras o palabras clave más importantes de los documentos para estudiar la estructura conceptual. Otra de las técnicas para construir el mapeado científico es la co-citación (McCain,1990). Esta técnica utiliza los recuentos de co-citación para construir medidas de similitud entre documentos, autores o revistas. De manera más específica, Small (1973) la define como la frecuencia con la que dos unidades se citan juntas. Dentro de las técnicas de co-citación está la de documentos y la de autores (White y Griffith, 1981). La co-citación de autores según Zhao (2006) consiste en la frecuencia con la que el nombre de dos autores A y B son citados dentro de un documento. Mientras que Sanguri et al., (2020) definen la co-citación de documentos como el número de veces que dos documentos son citados de manera conjunta en un documento . Asimismo, White y Griffith (1981) aseguran que la cocitación de documentos conecta documentos específicos publicados, guardando una estrecha relación con la co-citación de autores ya que permite la conexión de cuerpos de escritos de una persona. Ambas técnicas permiten la creación de mapas de conocimiento. Siguiendo con el estudio de las técnicas que permiten la realización de los mapas de conocimiento destaca el análisis de coautores. Esta metodología examina las redes sociales que crean los científicos al colaborar en artículos (Acedo et al.,

Análisis de la producción científica en el uso de la realidad virtual en la educación a partir de la estructura conceptual, social e intelectual. Gonzalo Lorenzo Lledó

Página $\mathbf{3}$ de $\mathbf{3 1}$ 
2006).Finalmente, Martínez et al., (2015) inciden en la coautoría como medida de colaboración que se basa en el precepto de que ser coautor de una publicación es sinónimo de ser responsable del trabajo realizado.

La utilización de la aproximación bibliométrica basada en mapeado científico junto con los indicadores de rendimiento bibliométrico supera algunos de los problemas que presentan los métodos tradicionales de la literatura científica como son las revisiones estructuradas de la literatura y el metaanálisis (Zupic y Cater, 2015). Estos autores expones que el mapeado evita los problemas de sesgo del investigador y el número limitado de trabajo cuando se trabaja con la revisión estructura de la literatura. En esta línea Zupic y Cater (2015) manifiestan que el mapeado supera los problemas del metaanálisis relacionado con el tipo de estudio que se trabaja y la amplitud de estos. Por otro lado, Martínez et al., (2015) sostienen que el mapeo científico proporciona nuevos hallazgos e información importante sobre la dinámica del campo de estudio como disciplina científica. Por ejemplo, cómo se ha formado la base de conocimiento, cuáles son sus fortalezas de conocimiento científico y qué temas podrían ser objeto de estudio en el futuro. Asimismo, el mapeado también permite mostrar la evolución conceptual, intelectual de un campo de investigación, descubriendo patrones, tendencias, estacionalidad y valores atípicos (Martínez et al., 2015). Para profundizar en el estudio de cualquier campo de conocimiento es necesario definir tres conceptos que son la estructura conceptual, la estructura intelectual y la estructura social. Donthu et al., (2021) definen la estructura conceptual a partir de la co-ocurrencia de palabras (Keywords Plus y Keyword authors) presentada en los diferentes documentos. Estos mismos autores concluyen que la estructura intelectual se define por la repetición de diferentes patrones de citación de fuentes, autores o documentos. Por último, Glänzel (2002) explica que la estructura social se puede representar mediante redes de colaboración en función de tres variables que son países, instituciones y autores. De esta forma se puede observar cómo se relacionan en el campo de conocimientos estos elementos.

El estudio planteado mediante el mapeado científico necesita una serie de técnicas de representación y análisis de la información para representar el conocimiento obtenido de la estructura conceptual, intelectual y social. Para ello utiliza la teoría de grafos y análisis de redes (Cuccurullo et al., 2016). En la teoría de grafos cada objeto se representa mediante un vértice y una relación de dos objetos se expresa mediante una arista (Rahman, 2017). Como resultado esta técnica se puede utilizar para modelar cualquier tipo de información que responda al esquema de objetos y relaciones entre ellos. De esta forma se pueden modelar los procesos de co-citación y co-palabras. Por tanto, según Sporns (2018) la correcta aplicación de la teoría de grafos puede proporcionar nuevos e importantes conocimientos sobre la estructura, la evolución y el desarrollo de los campos de conocimiento. Por otro lado, para Zhao et al., (2021) la teoría de redes intenta representar un conjunto de expertos académicos, información y conocimientos para analizar un tema especial. El objetivo es realizar un estudio estadístico sobre los mapas generados para mostrar diferentes medidas de relación o solapamiento de los diferentes cluster (Holden et al., 2005). Con este objetivo Donthu et al., (2021) plantean tres métricas de red que son utilizadas durante el estudio Page Rank, closeness centrality y betweness centrality. Pradhan y Pal (2020) definen closeness centrality como determinar la posición de un nodo respecto al resto de la red. Asimismo, el parámetro betweness centrality determina la frecuencia con la que un nodo es elegido por otro para realizar el

Análisis de la producción científica en el uso de la realidad virtual en la educación a partir de la estructura conceptual, social e intelectual. Gonzalo Lorenzo Lledó

Página 4 de 31 
camino. Mientras que el Page Rank establece el número de veces que un documento/autor es citado por los actores más importantes del campo (Goyal y Komar, 2021)

Tomando como referencia las teorías sobre bibliometría y mapeado científico, se exponen algunas investigaciones previas que se han llevado a cabo con el uso de estas técnicas. Los trabajos de Hamilton et al., (2021) realizan una revisión sistemática donde se examinó los estudios experimentales desde 2013, en los que se compararon los resultados cuantitativos del aprendizaje utilizando la RVI basada en HMD con métodos pedagógicos menos inmersivos. Los resultados muestran que la realidad virtual inmersiva facilita el aprendizaje de problemas altamente complejos que requieren comprensión y visualización especial. Estos autores también concluyen que esta tecnología favorece el desarrollo y adquisición tareas procedimentales además de la transferencia de habilidades virtuales a entornos reales. En este sentido Hamilton et al., (2021) aseguran el gran potencial de la RV como herramienta de enseñanza basado en pruebas teóricas y experimentales previas. En otras investigaciones Radianti et al., (2020) desarrollan un estudio del mapeo sistemático en el empleo en las tecnologías de RV en la enseñanza superior. Por ejemplo, se pudo observar que el interés por el uso de la RV en el campo educativo es cada vez mayor sin embargo se observa una ausencia de estudios concluyentes que hablen sobre sus beneficios. En cuanto a las tecnologías descritas Radianti et al., (2020) garantizan que las herramientas utilizadas son experimentales y su estudio se basaba en el rendimiento y usabilidad. De igual manera, se pudo observar que existen muy pocos trabajos que expliquen como adaptar la enseñanza basada en RV en el plan de estudios. En otros trabajos, Jensen y Konradsen (2018) tienen como objetivo evaluar sistemáticamente la calidad de los estudios y sintetizar y discutir sus resultados, centrándose especialmente en cómo la experiencia del alumno de RV afecta a los resultados del aprendizaje. Asimismo, Jensen y Konradsen (2018) aseguran que la RV es adecuada para enfoques y teorías de éxito, como el constructivismo, el aprendizaje activo o el aprendizaje basado en simulación. También estos autores identificaron situaciones las que la RV es útil para la adquisición de habilidades. Por ejemplo, las habilidades cognitivas relacionados con el recuerdo y la comprensión de la información, las habilidades psicomotoras relacionadas con el movimiento de la cabeza. Estos autores recomiendan más investigaciones rigurosas que examinen los usos más prometedores de los HMD en un contexto educativo o de formación auténtico

Por todo ello y debido al gran abanico de oportunidades que nos presenta el uso de la RV en el ámbito educativo es necesario analizar las tendencias que se producen en los estudios sobre el uso de esta herramienta en entornos de aprendizaje para tener una visión general de la comunidad científica para la elaboración de futuras líneas de investigación. En la línea de obtener conocimiento desde una perspectiva internacional, el objetivo de este estudio es analizar la evolución en la producción científica sobre el uso de la realidad virtual en la educación mediante la aproximación bibliométrica del mapeado científico a partir del estudio de la estructura conceptual, social e intelectual del campo de conocimiento. Para guiar la implementación de este estudio se plantean las siguientes preguntas de investigación.

1. ¿Cuál es la estructura conceptual de las fuentes que publican documentos sobre el uso de la realidad virtual en la educación?

Análisis de la producción científica en el uso de la realidad virtual en la educación a partir de la estructura conceptual, social e intelectual. Gonzalo Lorenzo Lledó

Página 5 de $\mathbf{3 1}$ 
2. ¿Cuál es la organización intelectual y social de los autores en el campo de la realidad virtual en la educación?

3. ¿Cuál es la organización social de los países de los autores en el campo de estudio?

4. ¿Cuál es la estructura intelectual de los documentos publicados en el campo de estudio?

5. ¿Cuál es la estructura conceptual de los documentos analizados?

\section{2.- Método.}

Para responder a las preguntas de investigación se ha escogido entre las diversas técnicas bibliométricas el flujo de trabajo estándar para la cartografía científica que establecieron Aria y Cuccurullo (2017). Se ha seleccionado esta técnica porque según RodríguezGarcía et al., (2019) permite evaluar y estimar la calidad, productividad y evolución científica de un determinado campo o tema de investigación. Para Aria y Cuccurullo (2017) existen cinco fases para la aplicación del flujo de trabajo estándar para la cartografía científica y que se indica en la figura 1

\section{Figura 1.}

\section{Flujo estándar para la cartografía científica.}

Se definen las preguntas de investigación y se escogen cuáles son los métodos bibliométricos apropiados para dar respuesta. Se debe escoger el periodo de tiempo específico donde realizar el estudio.
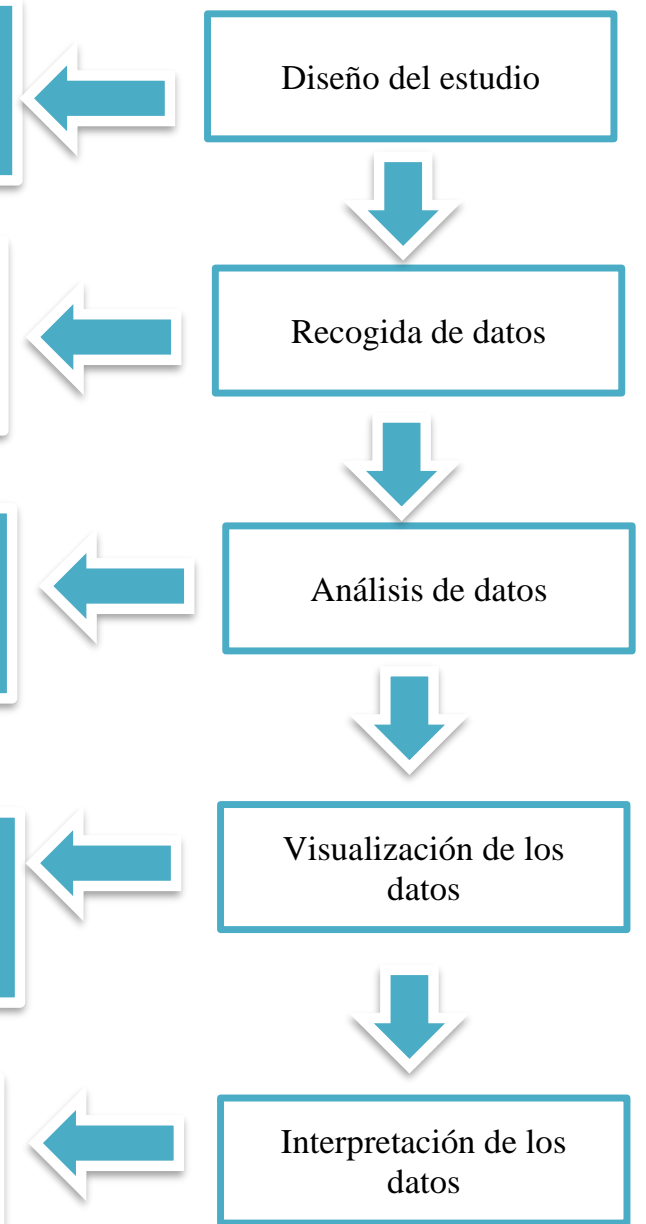

Se debe escoger el software bibliométrico para desarrollar el análisis de los datos. Una de las técnicas estadísticas que se utiliza es el análisis de redes junto con metodologías como la co-citación y co-palabras.

En esta fase se determina la forma en la que se va a mostrar el mapeado científico. Por ejemplo en la herramienta bibliometrix se escoge la variable que se quiere representar, el número de nodos, el algoritmo de clustering, además del diseño de la red.

La interpretación de los datos se lleva a cabo a partir de una serie de parámetros por ejemplo el Page Rank, closeness o el btw centrality
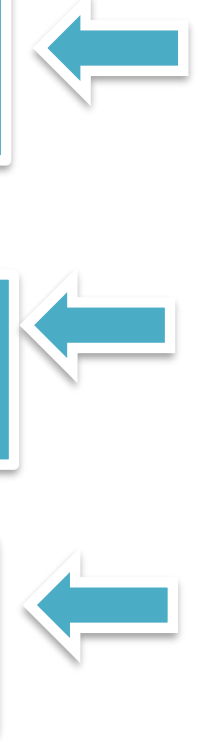
datos

Análisis de la producción científica en el uso de la realidad virtual en la educación a partir de la estructura conceptual, social e intelectual. Gonzalo Lorenzo Lledó 
Finalmente, el estudio ha sido implementado teniendo en cuenta tres niveles de análisis siguiendo lo expuesto por Rodríguez-Soler et al., (2020): fuentes, documentos y autores

\section{1.- Diseño y procedimiento}

En la primera fase de la investigación se lleva a cabo el diseño del estudio. En ella es necesario que se establezca cuáles son las preguntas de investigación. Según Aria y Cuccurullo (2017) son de tres tipos. La primera tiene como objetivo establecer una base de conocimiento dentro de un campo de investigación. La segunda y la tercera son el punto de partida para la formulación de las preguntas de investigación de nuestro estudio ya que tiene como finalidad la estructura la estructura intelectual y la evaluación de la estructura conceptual, mientras que la tercera profundiza en la producción de una estructura de red social de una comunidad científica determinada. Como técnicas bibliométricas se escogieran la co-citación y las co-palabras a partir de lo indicado en el apartado 1.1 .

La segunda fase del flujo estándar para cartografía científica establecida por Aria y Cuccurullo (2017) es la recogida de datos. Para conseguir este objetivo resulta necesario determina la base de datos con la que se va a trabajar. Se ha escogido la Colección Principal de la Web of Science. Esta base de datos ya ha sido utilizada en estudios referidos a otros campos como por ejemplo Diem y Wolter (2013). Estos autores investigan la idoneidad y solidez de los parámetros bibliométricos con el objetivo de medir el rendimiento de los investigadores en Ciencia de la Educación en suiza. Para ello utilizan la Web of Science y Google Scholar. Los resultados han mostrado por ejemplo una correlación positiva entre la producción referida al número de publicaciones y el resultado centrado en las citas, o calidad y cantidad de las publicaciones. Asimismo, estos autores afirman que es necesario establecer unos criterios claros en los estudios de manera que se evite incluir artículos con baja calidad científica. Esta se refiere a documentos publicados que se encuentren en revistas de poco prestigio o cuartiles bajos. En otra de las investigaciones Autor et al. (2020) utilizan Web of Science y Scopus para analizar la producción científica en la aplicación de la realidad virtual para la mejora de habilidades sociales en alumnos con Trastorno del Espectro Autista (TEA). Los resultados reflejan que la ausencia de estudios previos ha puesto en valor el uso del estas dos bases de datos para conseguir obtener una imagen sobre el uso de esta herramienta. Por otro lado, la Web of Science incluye información que no está presente en otras como el tipo de artículo, el índice de los autores, referencias bibliográficas o las direcciones institucionales (Repiso y Torres-Salinas, 2016). Además, asigna a cada documento más de una categoría temática lo que permite un mejor análisis y clasificación del material ( De Moya-Anegon et al., 2006; Herrero-Solana y Corera-Álvarez, 2016).

Tras la selección de la base de datos, se llevará a cabo la filtración de los documentos utilizando para ello la metodología PRISMA (Page et al., 2021). Esta herramienta presenta como principal función un desarrollo de la mejora de la claridad, calidad y consistencia sobre la información metodológica y los resultados obtenidos en las revisiones sistemáticas (Hutton et al., 2016). Esta protocolo facilita tanto la razón para llevar a cabo la revisión como el enfoque y en análisis planificado (Moher et al., 2015). Esta metodología no tiene su aplicación exclusiva en el ámbito clínico sino que se puede utilizar de manera transversal (Urrutia y Bonfill, 2010). Para la aplicación de esta

Análisis de la producción científica en el uso de la realidad virtual en la educación a partir de la estructura conceptual, social e intelectual. Gonzalo Lorenzo Lledó

Página $\mathbf{7}$ de $\mathbf{3 1}$ 
metodología es necesario tener en cuenta presente el sesgo que introduce la persona que realiza la selección de los documentos por este motivo se introdujo un segundo evaluador durante el proceso de esta manera se evita el sesgo de evaluación (Manterola, et al., 2013)

La filtración de todos los documentos se llevó mediante cuatro fases según lo indicado en PRISMA (Page et al., 2021). En la primera fase llamada de identificación se implementó la búsqueda inicial de los documentos. Para dotar la búsqueda de un rigor científico las palabras clave fueron extraídas del Tesauro de ERIC y UNESCO. Según Haas et al., (2020) en estas bases aparecen los términos más utilizados en los artículos reconocidos por la comunidad científica. Los términos fueron:

Virtual reality, Immersive Virtual Reality, Desktop Virtual Reality, Head Mounted Display, Infant Education, Primary Education, Secondary Education, Education, Higher Education, High School.

La introducción de las palabras seleccionadas en el código booleano dio las siguientes líneas de código.

TOPIC: ((("Virtual reality" OR "Immersive virtual reality" OR "Desktop Virtual Reality" OR "Head Mounted Display") AND ("Infant Education" OR " Primary Education" OR "Secondary Education" OR "Education" OR "Higher Education" OR "High School" ))) OR TITLE: ((("Virtual reality" OR "Immersive virtual reality" OR "Desktop Virtual Reality" OR "Head Mounted Display") AND ("Infant Education" OR " Primary Education" OR "Secondary Education" OR "Education" OR "Higher Education" OR "High School" ))

Los resultados de la fase 1 dan un total de 4961 documentos. En la segunda fase, denominada "Screening" se aplicaron los criterios de Inclusión (IC1, IC2, IC3) y los criterios de exclusión (EX1, EX2, EX3). En la tabla 1 se recogen todos los criterios de inclusión y exclusión aplicados en esta fase y en las siguientes

\section{Tabla 1.}

Criterios de inclusión y exclusión

\begin{tabular}{ll}
\hline \multicolumn{1}{c}{ Criterios de inclusión } & Criterios de exclusión. \\
\hline IC1: Periodo 1996-2019 & EX1: Presencia de documentos duplicados. \\
IC2: Categorías de la Colección Principal de la & EX2: No acceso a los documentos. \\
Web of Science. Education and Educational & \\
$\begin{array}{l}\text { Research, Education Science Disciplines. } \\
\text { IC3: Lenguaje: Inglés y Castellano }\end{array}$ & EX3: Exclusión de documentos del tipo Letter, \\
& Book Chapter, Editorial Material y News Item. \\
IC4: Tipo de estudio: Revisiones e intervenciones. & EX4: Tipo de tecnología. \\
IC5: Temática de realidad virtual en Educación. & EX5: Edad de los participantes. \\
\hline
\end{tabular}

En la tercera fase se realiza un análisis temático de los contenidos mediante la fase de "Elegibilidad". Se aplicaron los criterios de inclusión y exclusión (IC4, IC5, EX4, EX5). En la última fase, denominada "inclusión", se especifica el tamaño final de la muestra, $\mathrm{n}=1163$. 
Asimismo, se añade una sabana reflejada en la tabla 2 sobre la información referida a la muestra obtenida.

\section{Tabla 2}

Características de la muestra.

\begin{tabular}{|c|c|c|}
\hline $\begin{array}{c}\text { Información principal sobre los } \\
\text { datos. } \\
\end{array}$ & Tipos de documentos. & Autores. \\
\hline Periodo 1996-2019 & Artículos de revista. 529 & Autores. 3523 \\
\hline Fuentes. 409 & Artículos de congreso. 594 & $\begin{array}{l}\text { Aparición de los } \\
\text { autores. } 4176\end{array}$ \\
\hline Documentos. 1163 & Revisiones. 30. & Autores de documentos \\
\hline $\begin{array}{l}\text { Promedio de años desde la publicación. } \\
6.61\end{array}$ & Artículos de early access. 10 . & de autoría única. 163 \\
\hline Media de citas por documento. 14.3 & Contenido del documento. & $\begin{array}{l}\text { Autores de documentos } \\
\text { con varios autores. } \\
3360\end{array}$ \\
\hline \multirow{2}{*}{$\begin{array}{l}\text { Promedio de cita } \\
\text { documento. } 1.586 \\
\text { Referencias. } 26055\end{array}$} & $\begin{array}{l}\text { Keyword Plus. } 990 . \\
\text { Author Keyword. } 2617\end{array}$ & $\begin{array}{l}\text { Colaboración de } \\
\text { autores. }\end{array}$ \\
\hline & & $\begin{array}{l}\text { Documentos de un solo } \\
\text { autor. } 174 \\
\text { Documentos por autor. } \\
0.336 \\
\text { Coautores por } \\
\text { documento.3.52 } \\
\end{array}$ \\
\hline
\end{tabular}

Mientras que en que en la figura 2 se muestra el diagrama de flujo que se ha implementado siguiente la declaración PRISMA. La tercera y cuarta fase del método propuesto por Aria y Cuccurullo (2017) referidos al análisis y visualización de los datos se explica en detalle en el apartado 2.3. Finalmente, el apartado referido a la interpretación de los datos su definición y justificación se ha desarrollo en el apartado 1.1.

Análisis de la producción científica en el uso de la realidad virtual en la educación a partir de la estructura conceptual, social e intelectual. Gonzalo Lorenzo Lledó 


\section{Figura 2.}

Diagrama de flujo según la declaración PRISMA
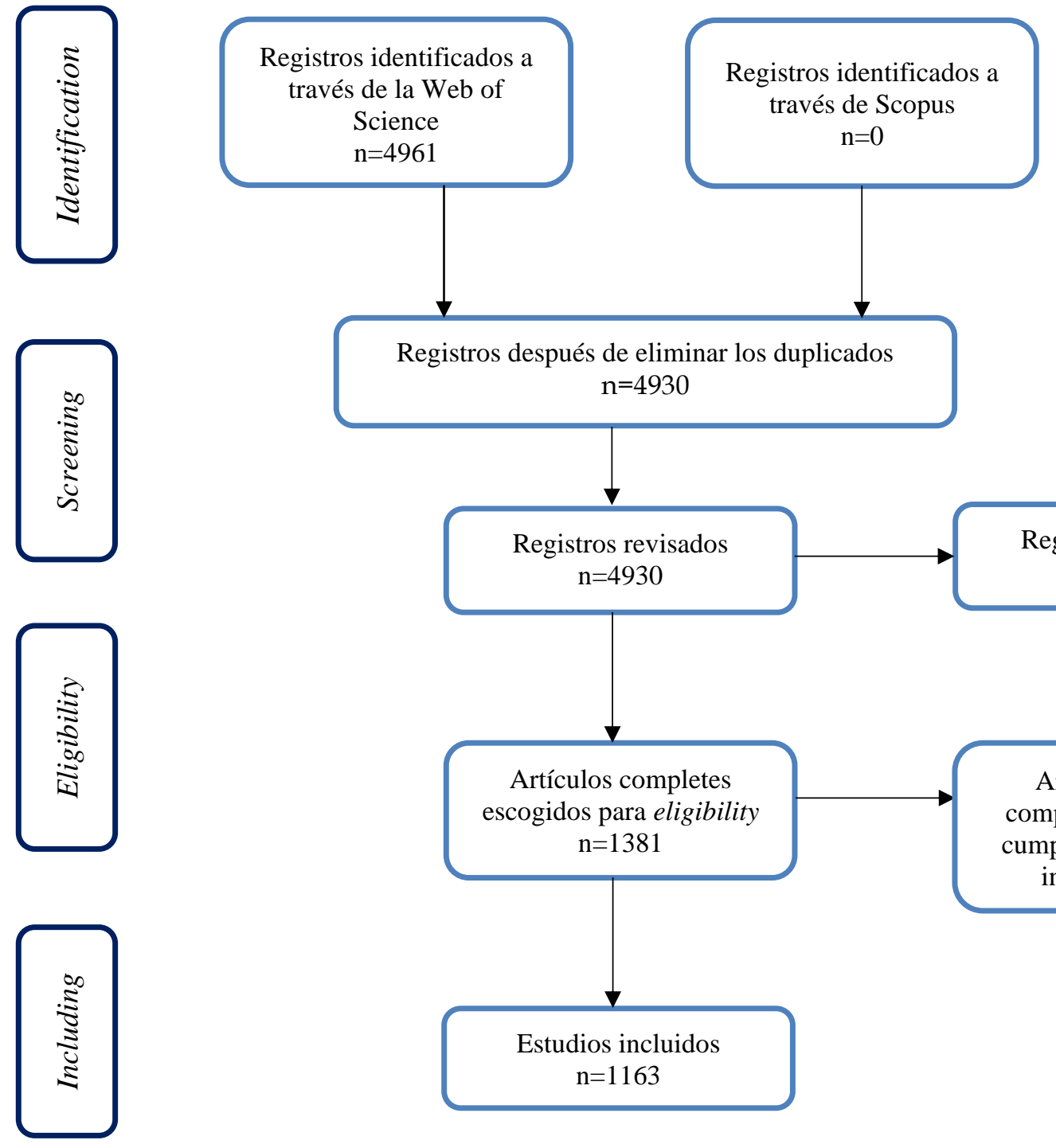

egistros después de eliminar los duplicados $\mathrm{n}=4930$
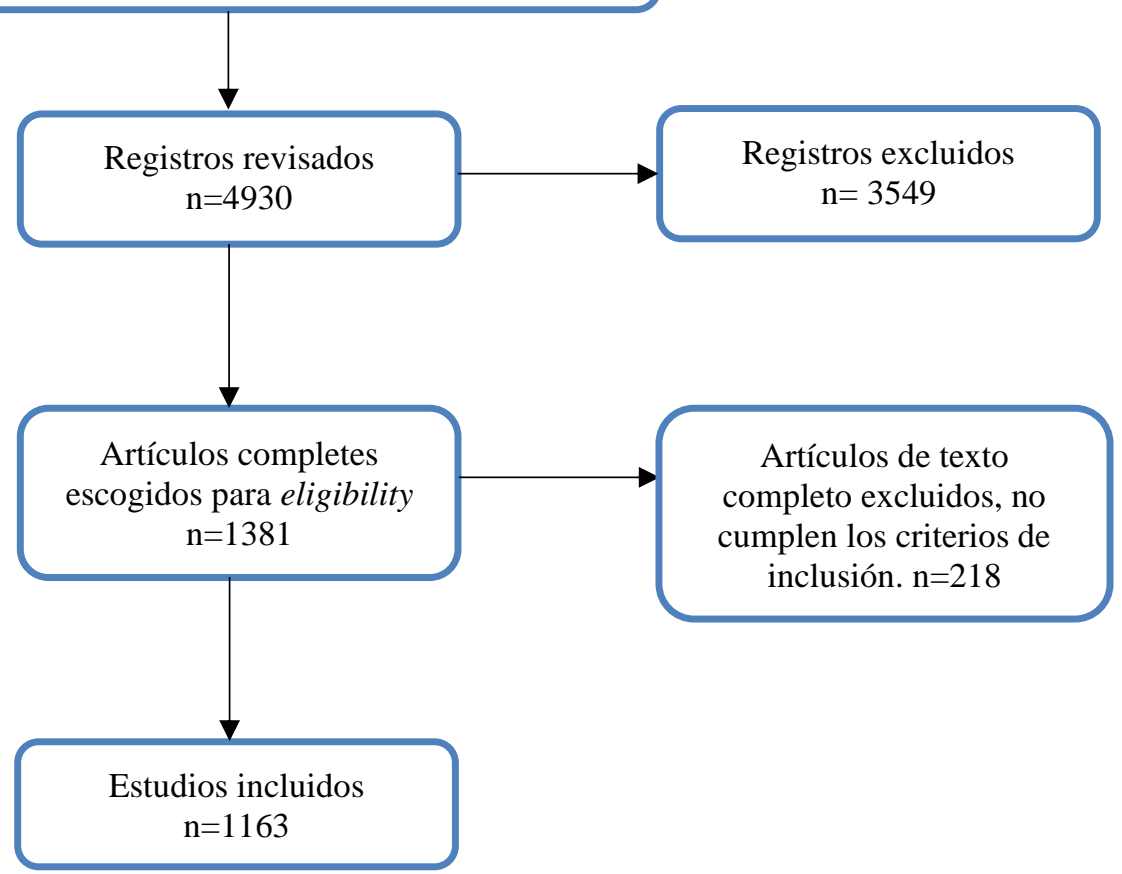

\section{3.- Análisis de datos.}

El análisis de los datos se llevará a cabo con bibliometrix que es una librería de R (Aria y Cuccurullo, 2017). Este software trabaja mediante los tres pasos establecidos en el mapeado científico: recopilación de datos, análisis de datos y visualización de datos. El análisis de los datos se desarrollará en función a una serie de métricas y unidades de análisis que se combinaran con técnicas bibliométricas. Esto queda reflejado a continuación en los tres niveles de estudio basados en el estudio de Rodríguez-Soler et al. (2020). 
1. Nivel de análisis: Fuentes, Métricas: dinámica de las fuentes y fuentes más productivas, unidades de análisis: revista, técnicas bibliométricas utilizadas: co-citación, técnicas estadísticas: redes, estructura: conceptual.

2. Nivel de análisis: autores, Métricas: autores más productivos y producción anual por autor junto con países más productivos, unidades de análisis: autores y países, técnicas bibliométricas: co-citación y colaboración, técnicas estadísticas: redes, estructura: intelectual y social.

3. Nivel de análisis: documentos, métricas: documentos más citados junto con Keyword authors-Plus, unidades de análisis: referencias, keyword autor/Plus, técnicas bibliométricas: co-palabras y co-citación, técnicas estadísticas: redes y mapas temáticos junto con evolución temática, estructura: intelectual y conceptual.

Toda la información referida a los algoritmos de clustering y de clustering layout deben ser ajustados en el entorno de $\mathrm{R}$ junto con los nodos de colaboración y co-citación. Esta información queda recogida en la tabla 3

\section{Tabla 3}

Parámetros de configuración de $R$ en las redes de Co-citación y colaboración basado en Rodríguez-Soler et al., (2020).

\begin{tabular}{|c|c|c|c|c|c|}
\hline \multirow[t]{2}{*}{ Redes } & \multicolumn{3}{|c|}{ Co-Citation } & \multicolumn{2}{|c|}{ Colaboración } \\
\hline & Fuentes & Autores & Referencias & Autores & Países \\
\hline Clustering & Walktrap & Walktrap & Walktrap & Walktrap & - \\
\hline Nodos & 50 & 50 & 50 & 50 & 50 \\
\hline Min Ejes & 2 & 2 & 2 & 2 & 2 \\
\hline N. ${ }^{\circ}$ Etiquetas & 50 & 50 & 50 & 50 & 50 \\
\hline Clúster Layout & Kamada y Kawai & Walktrap & Kamada y Kawai & Kamada y Kawai & - \\
\hline
\end{tabular}

Los algoritmos que se han utilizado han sido el clustering denominado Walktrap y diseñado por Pons y Latapi (2006). La gran ventaja respecto a otros algoritmos es capacidad para capturar información y detectar comunidades en una red. Asimismo, propone una nueva distancia entre dos vértices a partir de diversos caminos aleatorios. Por otro lado, destaca como algoritmo de clustering layout el de Kamada y Kawai (1989). En este algoritmo se intenta obtener la disposición óptima de los vértices. Esto se consigue mediante un sistema de energía de muelles siendo la distancia entre los vértices como un muelle virtual.

Análisis de la producción científica en el uso de la realidad virtual en la educación a partir de la estructura conceptual, social e intelectual. Gonzalo Lorenzo Lledó 


\section{3.- Resultados.}

\section{1.- Fuentes}

El primero de los niveles de análisis son las fuentes. Con el objetivo de poder complementar la información proporcionada por el mapeado científico es necesario profundizar en algunas métricas de rendimiento. La primera de ellas es el estudio de producción de las fuentes. Para ello, se divide el periodo de estudio en dos: el primero de 1994-2008 mientras que el segundo tiene lugar de 2009-2019. En ambos periodos la revista Computers y Education es la revista que mayor número de documentos produce y la que recibe mayor cantidad de citas, seguida de Engineering Education y de las publicaciones de medicina Journal of Surgical Education y Anatomical Science Education. Las tres primeras tienen los valores de h (índice de Hirsch) más elevados con 39, 21 y 20 respectivamente. Este índice según, Costar y Bordons (2007) representa el número h de documentos que tienen al menos h citas cada uno. Estos autores concluyen que para su cálculo es necesario ordenar las publicaciones en orden descendente del número de citas. De manera que se permite identificar el punto en el que el número de orden coincide con el número de citas que ha recibido el documento. Una de las revistas que no está entre las más productivas, pero recibe muchas citas es Medical Education. De igual manera, la dinámica de las fuentes nos muestra como en el periodo 2014-2018 son las revistas de medicina las que copan las posiciones más altas de productividad.

La red de co-citación de las revistas está formada por dos clústeres. Un clúster según Ezugwu et al., (2020), consiste en la agrupación de objetos que tienen características o atributos similares. En la investigación planteada, los clústeres están formados por aquellos documentos que tienen citas de fuentes en común entre ellos. El primero de los clústeres de la red contiene 14 revistas, destacando Medical education con una puntuación de Btw centrality de 13.28. En este sentido, el valor de closeness oscila entre 0.013-0.020. Mientras que el segundo está formado por 36 revistas recibiendo la mejor valoración Computers y Education con un Btw centrality de 23.70, ocupando el segundo lugar British Journal of Educational Technology con 6.04. De igual manera, el valor de closeness del clúster oscilan entre 0.016-0.020.Este segundo clúster se centra más en temáticas de Tecnología y Educación por el contrario el primero está más relacionado con Tecnología en la medicina. Además, se puede observar cómo existen revistas que a pesar de no estar entre las más productivas y las más citadas presentan un gran reconocimiento dentro del campo. En la tabla 4 se muestran los valores de Btw y closeness de las revistas más importantes.

\section{Tabla 4}

Valores de btw y de closeness de las revistas más importantes en la red de co-citación

\begin{tabular}{cccc}
\hline Revista & Btw & Closeness & Clúster. \\
\hline Medical Education & 13.28 & 0.019 & 1 \\
Academic Medicine & 11.93 & 0.018 & 1 \\
Medical Teaching & 9.01 & 0.018 & 1 \\
Computers \& Education & 23.70 & 0.020 & 2 \\
$\quad$ British Journal of & 6.04 & 0.018 & 2 \\
Education Technology & & & \\
Computers in Human & 5.14 & 0.019 & 2 \\
$\quad$ Behavior & & & \\
\hline
\end{tabular}

Análisis de la producción científica en el uso de la realidad virtual en la educación a partir de la estructura conceptual, social e intelectual. Gonzalo Lorenzo Lledó 
Mientras que en las figuras 3 y 4 se observa la distribución en clústeres de las redes de co-citación de las revistas.

\section{Figura 3}

Redes de co-citación entre revistas. Clúster 1. Color rojo.

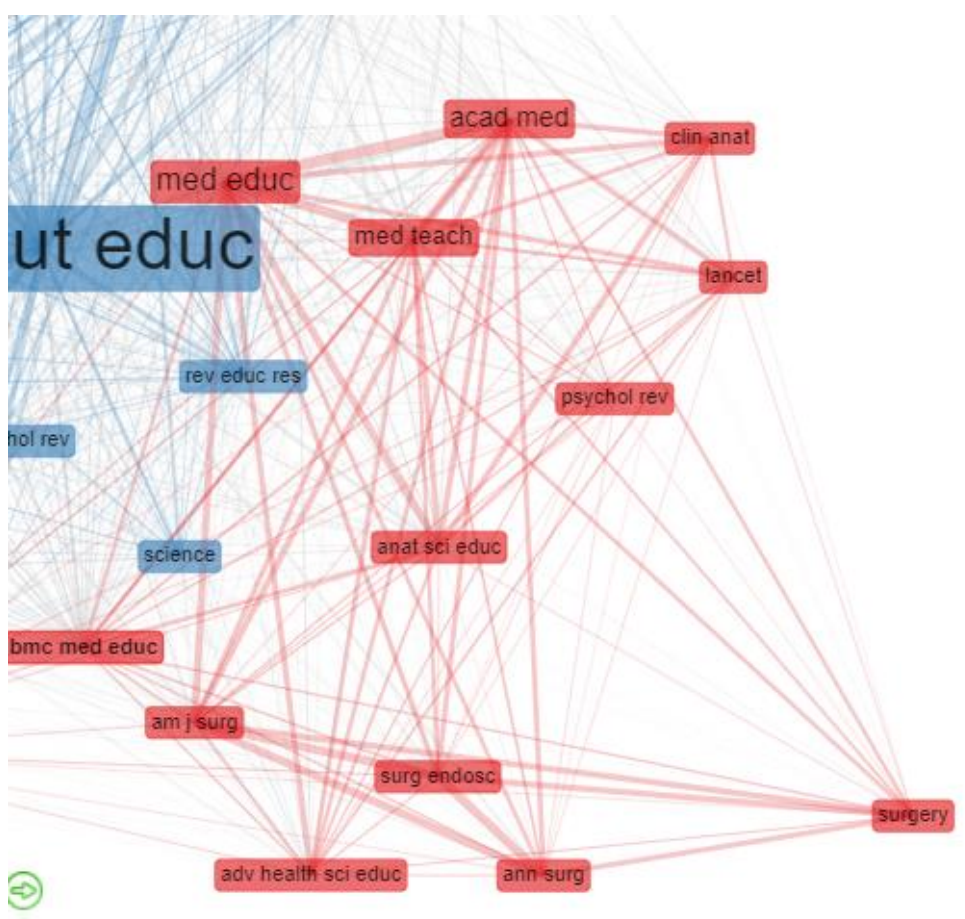

\section{Figura 4}

Redes de co-citación entre revistas. Clúster 2. Color azul.

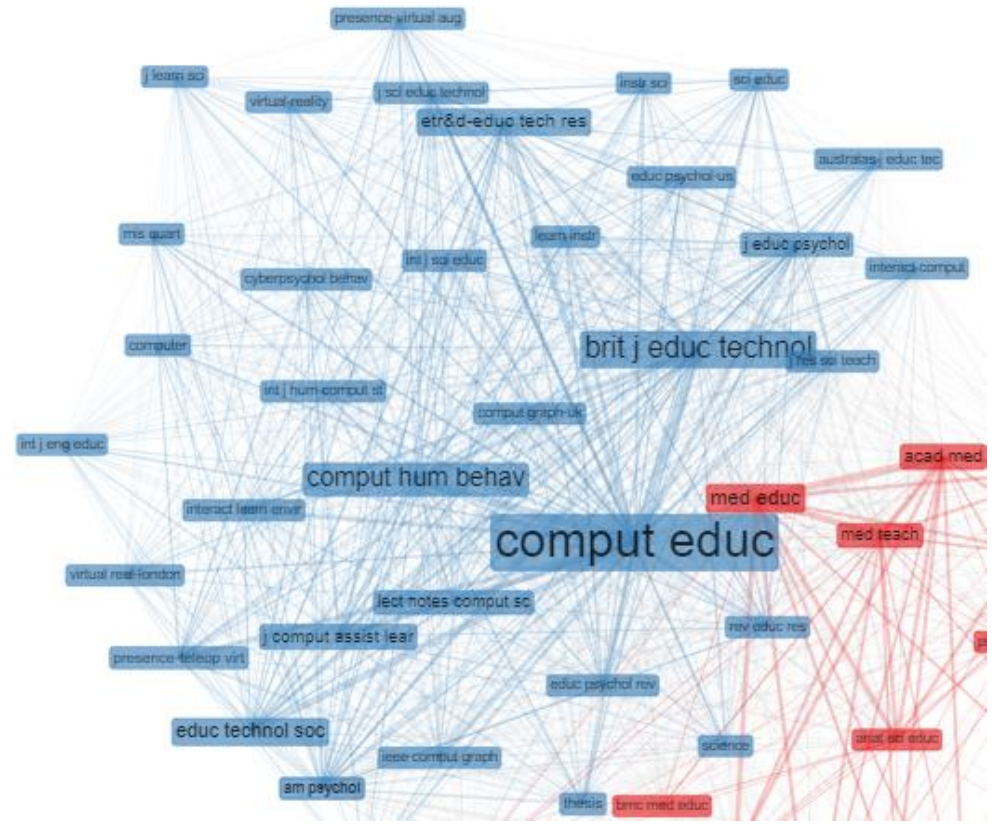

Análisis de la producción científica en el uso de la realidad virtual en la educación a partir de la estructura conceptual, social e intelectual. Gonzalo Lorenzo Lledó 


\section{2.- Autores.}

\subsection{1.- Autores más productivos, redes de colaboración y co-citación}

El campo de estudio está compuesto por 3455 autores, entre los cuales destacan cinco de los autores más productivos Kovas, Palkova o Wilson. En este sentido, los autores de producción única ocupa el $13.84 \%$ del campo de estudio. Siendo Szymczykt el que dispone de una mayor autoría de documentos fraccionados. En relación con las redes de colaboración entre autores, actualmente existe un total de 20 clúster (figura 5). Los clústeres más numerosos son el 1 y el 12 con redes de trabajo de 7 autores. En el primer clúster el autor más importante es Palkova con un valor btw de 8. Además, tres de los autores más productivos que son Palkova, Kovak y grivokostopoulou forman parte del primer clúster. Por el contrario, otros autores como Wilson forman parte de un clúster en solitario. De igual manera, la gran mayoría de las redes están formadas por equipos de tres a cinco autores. Mención especial recibe el clúster 12 donde existe un nexo bastante fuerte entre sus autores debido a valores closeness solo del $10 \%$.

\section{Figura 5}

Redes de colaboración entre autores.

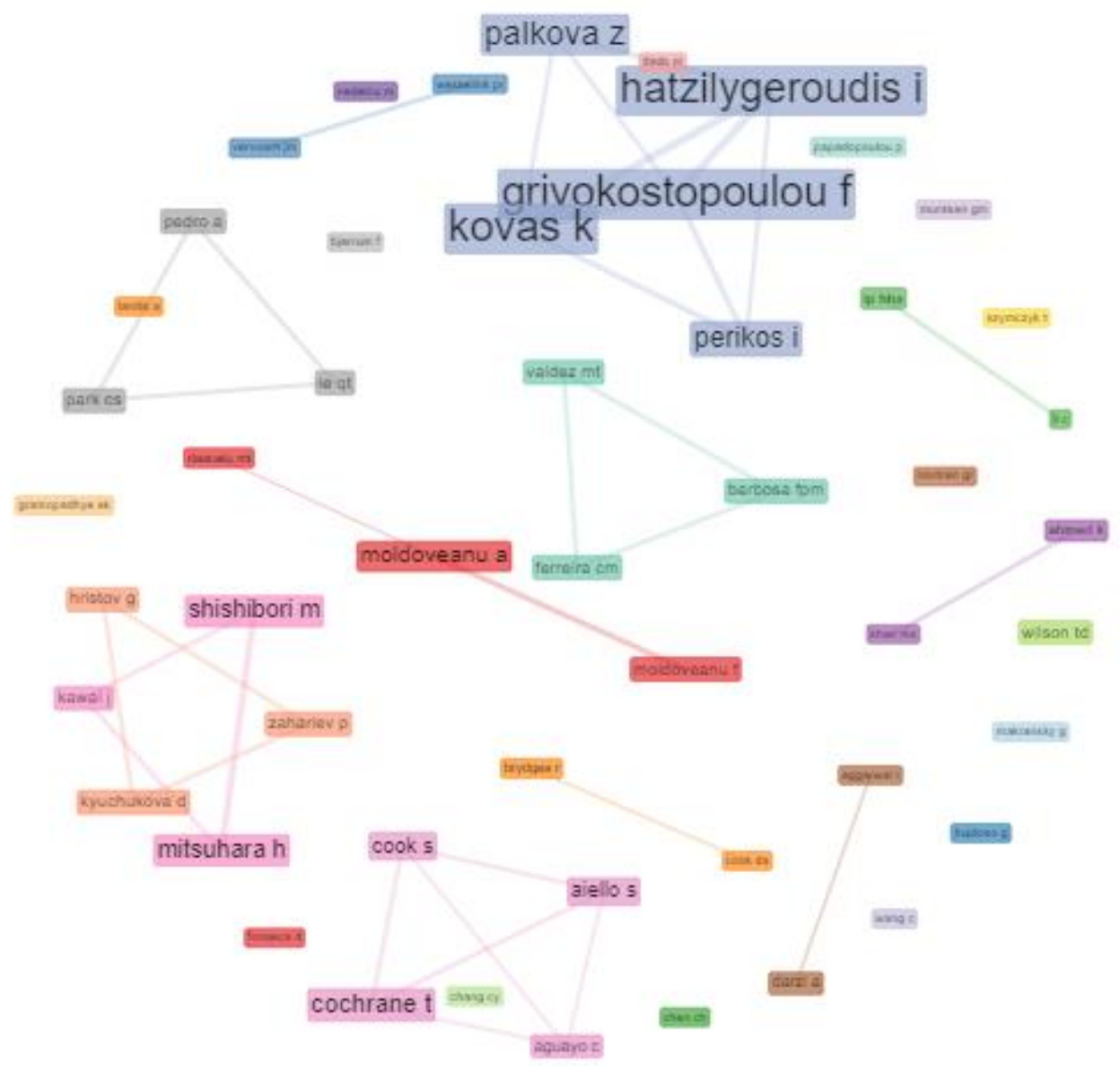

Análisis de la producción científica en el uso de la realidad virtual en la educación a partir de la estructura conceptual, social e intelectual. Gonzalo Lorenzo Lledó 
Las redes de co-citación entre autores hace posible la obtención de la imagen intelectual del campo de trabajo. En las figuras 6,7,8 se observa que existen 3 clúster de co-citación, representados por tres colores diferentes (rojo, azul y verde). El primero de ellos está formado por 18 autores, siendo Mayer, Re y Nicholson. Dt los que tienen los valores de bwt más elevados con 109.93 y 69.21 respectivamente. Asimismo, Mayer es el que recibe el mayor número de citas, pero no se encuentra entre los más productivos. En el caso de Nicholson ocupa la novena posición en cuanto al número de citas. En cuanto al segundo clúster lo componen 27 autores, siendo los que reciben un mayor reconocimiento Merchant, Z., y Dalgarno, b. con valores de Bwt de 55 y 41 . Ambos autores ocupan la segunda y décima posición como los más citados. Por último, el tercer clúster lo componen solo 5 autores. Los que más importantes son Seymour, con un valor de 10.01 y Aggarwal, r. con 10.65. Ninguno de estos dos autores se encuentra entre los más productores ni los más citados.

\section{Figura 6.}

Redes de co-citación entre autores. Clúster 1. Color rojo.

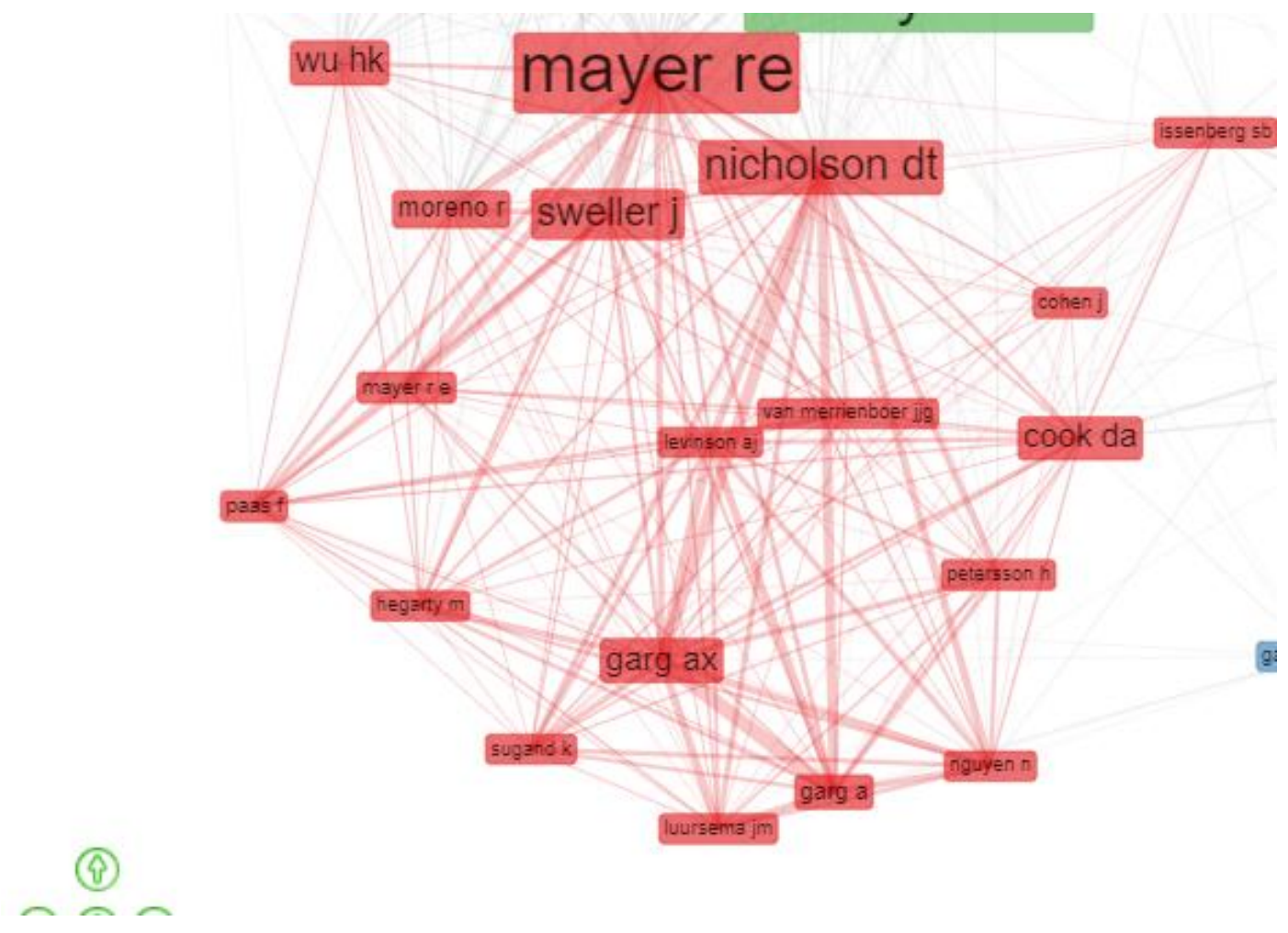

Análisis de la producción científica en el uso de la realidad virtual en la educación a partir de la estructura conceptual, social e intelectual. Gonzalo Lorenzo Lledó 
RED. Revista de Educación a Distancia. Núm. 69, Vol. 22. Artíc. 6, 30-Ene-2022

DOI: http://dx.doi.org/10.6018/red.502601

\section{Figura 7}

Redes de co-citación entre autores. Clúster 2. Color azul.

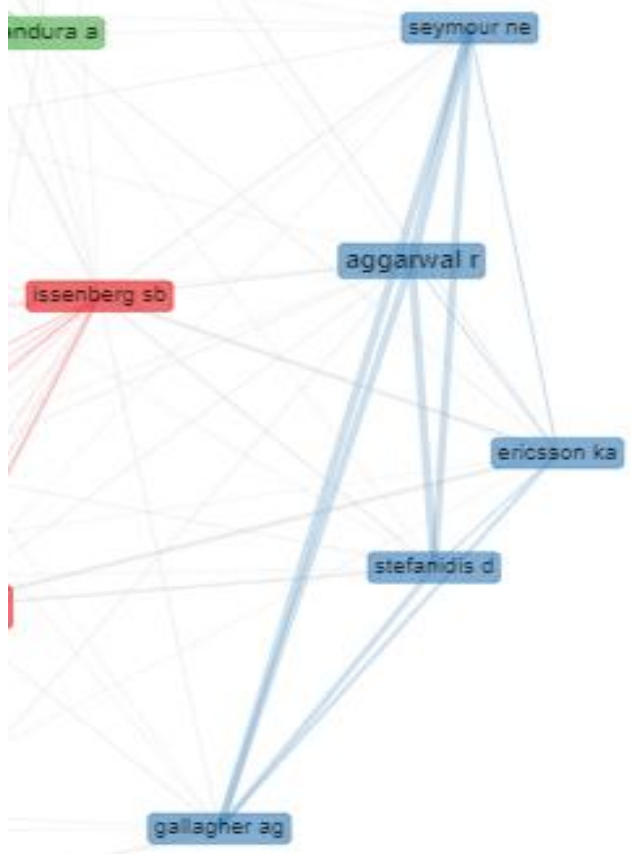

Análisis de la producción científica en el uso de la realidad virtual en la educación a partir de la estructura conceptual, social e intelectual. Gonzalo Lorenzo Lledó

Página 16 de 31 


\section{Figura 8}

Redes de co-citación entre autores. Clúster 3. Color verde.

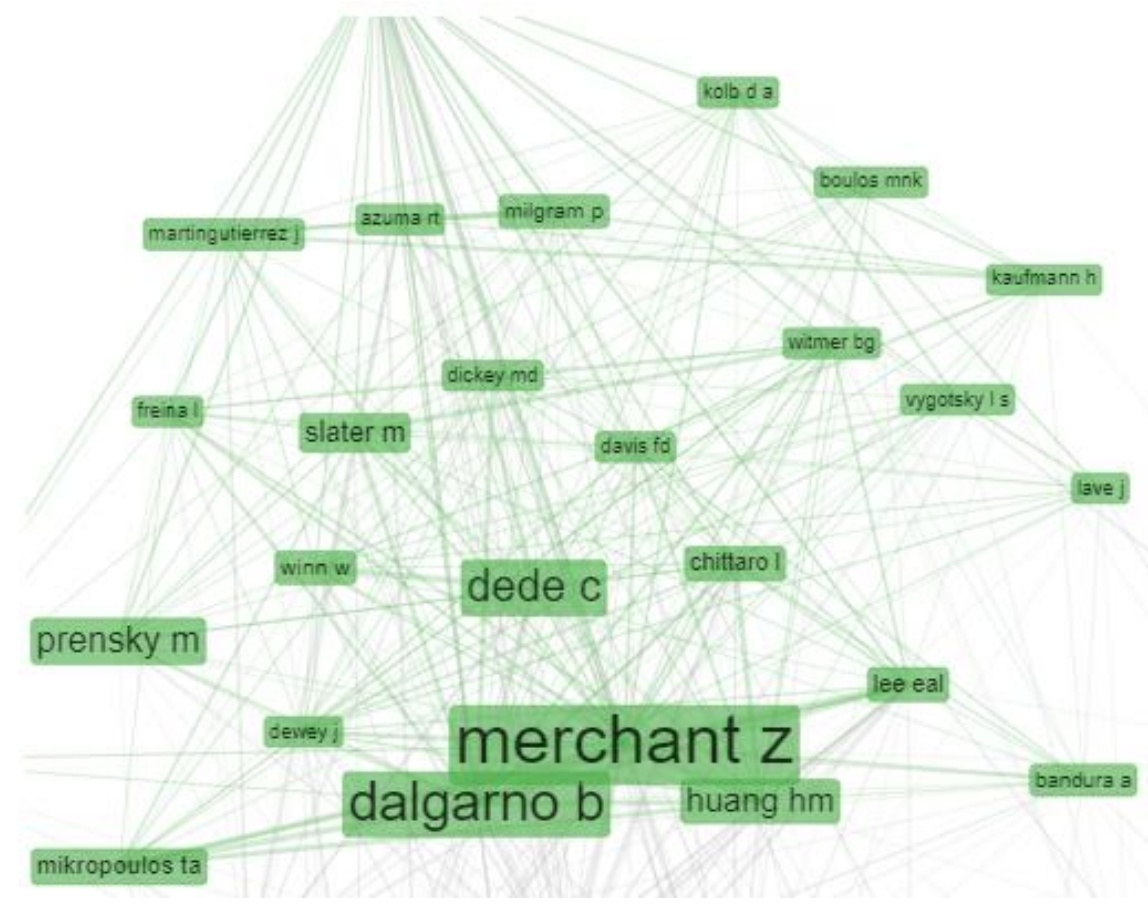

\subsection{2.- Producción, citación y redes de colaboración entre países.}

En cuanto a la producción científica de la temática, la primera posición está ocupada por Estados Unidos con 468 documentos, seguida de China y Canadá con 286 y 162 documentos. En este sentido Estados Unidos también ocupa la primera posición en el número de citaciones con 6151 seguida de Inglaterra con 1946 y de China con 1931. Por el contrario, es Serbia el país cuyos artículos reciben una mayor media de citaciones con 80, seguida de Suecia con 31.4 citas por artículo y de Inglaterra con 30.4. Toda esta información queda recogida en la siguiente tabla.

\section{Tabla 5}

Países más productores, número de citas y media de citas por documento.

\begin{tabular}{cccccc}
\hline País & Total, de Citas & País & $\begin{array}{c}\text { Media de citas } \\
\text { por } \\
\text { documento }\end{array}$ & País & $\begin{array}{c}\text { Número de } \\
\text { documentos }\end{array}$ \\
\hline Estados Unidos & 6151 & Serbia & 80 & Estados Unidos & 468 \\
Inglaterra & 1946 & Suecia & 31.4 & China & 286 \\
China & 1931 & Inglaterra & 30.4 & Canadá & 162 \\
Taiwan & 1230 & Estados Unidos & 29.01 & Inglaterra & 154 \\
Canadá & 985 & Emiratos Árabes & 25.4 & Taiwan & 126 \\
Australia & 724 & Corea & 23 & España & 122 \\
\hline
\end{tabular}

Análisis de la producción científica en el uso de la realidad virtual en la educación a partir de la estructura conceptual, social e intelectual. Gonzalo Lorenzo Lledó 
Por otro lado, es importante analizar dentro de este ámbito los valores MCP y SCP que significan según Rodríguez-Soler et al., (2020), para el primer caso documentos al menos uno de los autores no es nacional mientras que en el segundo todos los autores son nacionales. Para el caso que nos ocupa se puede ver como en USA el $89.20 \%$ de los documentos son de autoría autóctona (SCP) al igual que ocurre en China, en tercera posición Inglaterra tiene un $85.4 \%$. Asimismo, esto se refleja en la tabla 6 .

\section{Tabla 6.}

Países y sus valores de SCP y MCP

\begin{tabular}{ccc}
\hline País & SCP & MCP \\
\hline Estados Unidos & $89.20 \%$ & $10.8 \%$ \\
"China & $89.20 \%$ & $10.8 \%$ \\
Inglaterra & $85.4 \%$ & $14.6 \%$ \\
España & $82.5 \%$ & $17.5 \%$ \\
Taiwan & $78.9 \%$ & $21.1 \%$ \\
Canadá & $77.3 \%$ & $22.7 \%$ \\
\hline
\end{tabular}

En cuanto a las redes de colaboración entre países, estas están organizadas en 26 clúster de los cuales el $77 \%$ son redes individuales mientras que el $23 \%$ están compuestas por más de un país. Los clústeres más numerosos son el 1 y el 6 que están compuestos por 11 y 8 países respectivamente. En el clúster 1 destacan USA y Canadá con valores de Btw de 275 y 60, además estos dos países son los que han realizado el mayor número de colaboraciones con 15. Estos dos países ocupan la primera y tercera posición en cuanto a la producción científica y además en el clúster 6 destacan Inglaterra y Australia con valores de 201.75 y 56. Asimismo, estos dos países tienen el mayor número de colaboraciones dentro de la red con 6 . Ocupando además la cuarta y séptima posición en países más productores con 148 documentos y 76.

\section{3.- Documentos}

Para poder analizar los documentos que han sido más citados, se ha utilizado el criterio de McDonald et al., (2000) que indica que se escogen aquellos documentos que tengan el número de citas mayor de 170. El documento que mayor número de citas recibe es el de Issenberg et al., (2005) con 1692 citas, seguido por el de Wu et al., (2013) con 541 citas y la tercera posición es para Merchant et al., (2014) con 352 citas.

En cuanto a las citas locales que reciben los documentos, es decir las citas que reciben desde otros papers de la misma colección, se puede ver como el artículo de Merchant et al., (2014) tiene 58 citas, mientras que la segunda posición la ocupa Nicholson et al., (2006) con 48 citas mientras que la tercera posición es para Huang et al., (2010) con 30 citas. De esta forma se puede ver como Keedy et al., (2011) que no se encuentra entre los más citados presenta un porcentaje de citas locales del $32.65 \%$. En esta línea NGuyen, N. (2009) presenta un valor del 32.50, siendo la tercera posición también para Nguyen et al., (2012) con $28.85 \%$. Esta información queda reflejada en la tabla 7.

Análisis de la producción científica en el uso de la realidad virtual en la educación a partir de la estructura conceptual, social e intelectual. Gonzalo Lorenzo Lledó 


\section{Tabla 7}

Cantidad de citas locales, globales y porcentaje entre ellas.

\begin{tabular}{cccc}
\hline Documento & $\begin{array}{c}\text { Citas } \\
\text { Globales }\end{array}$ & Citas Locales & \% Citas locales \\
\hline Issenberg et al. (2005) & 1692 & 21 & $1.24 \%$ \\
Wu et al. (2013) & 541 & 25 & $4.62 \%$ \\
Merchant et al.(2014) & 352 & 58 & $18.47 \%$ \\
Nicholson et al. (2006) & 195 & 48 & $25.13 \%$ \\
Huang et al. (2010) & 183 & 33 & $18.03 \%$ \\
\hline
\end{tabular}

Otra de las variables que esta intrínsecamente relacionada, es el análisis de la co-citación de documentos. Como se puede ver en la figura 9, 10 el campo de estudio está dividido en dos clústeres, representado en azul y rojo. El primero de ellos consta de 27 documentos, siendo el que tiene el valor de Btw más elevado Merchant et al., (2014) con 170.15. Este ocupa la tercera posición en cuanto al número de citas globales y el primero en citas locales. El segundo clúster está formado por 23 documentos. Al igual que el caso anterior, el que adquiere el mayor peso es uno de los más citados Nicholson et al., (2006) con un valor btw de 223.81 ocupando la posición 11 en la cantidad de citas globales y la segunda posición en citas locales. El valor de closeness es la red oscila entre 0.008 hasta 0.01 que hacen referencia a variaciones en torno al $25 \%$. Además, la red está separada en dos clústeres claramente diferenciados sin conexión entre clúster.

\section{Figura 9.}

Redes de co-citación entre documentos. Clúster 1. Color Rojo.

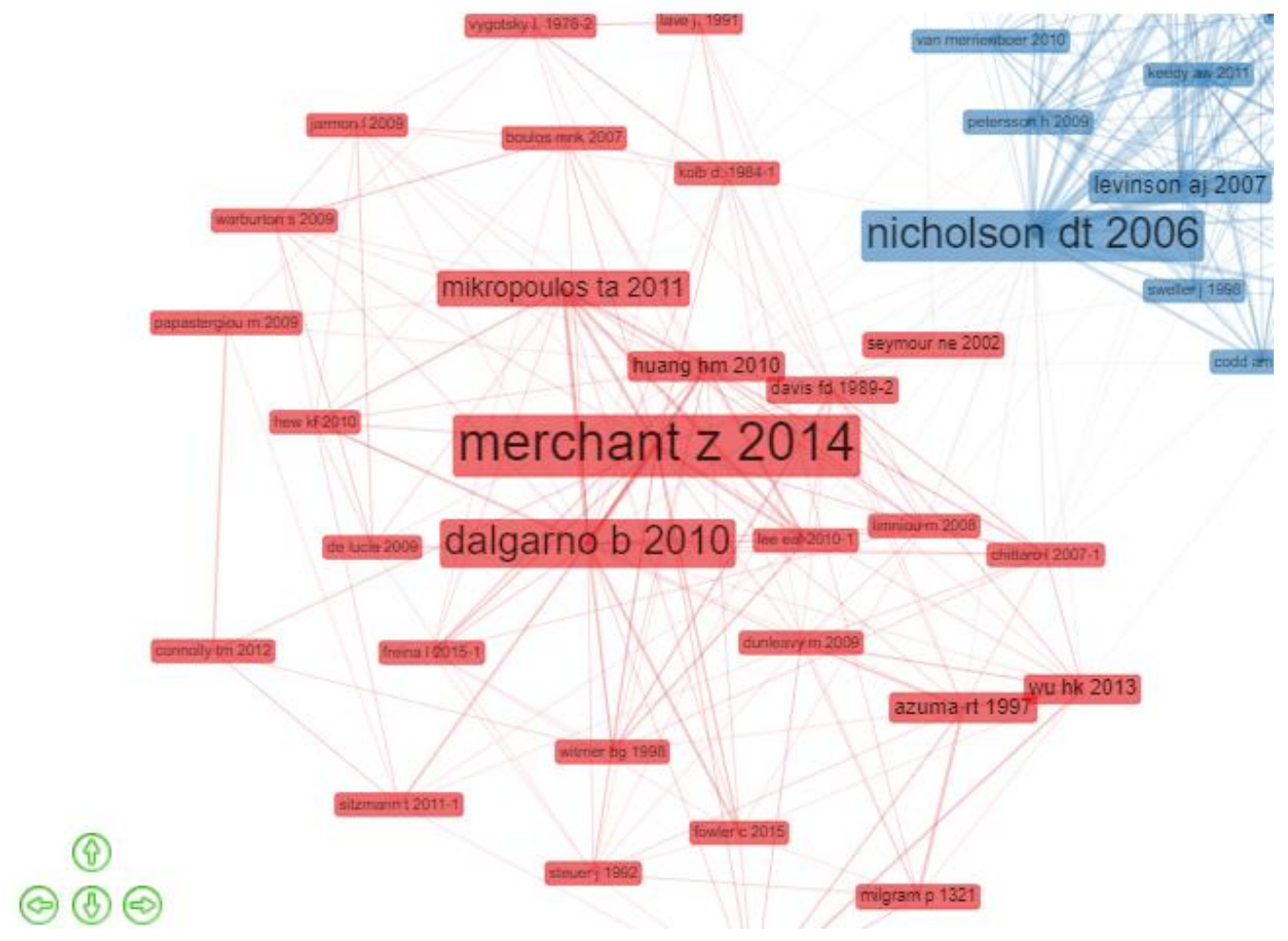

Análisis de la producción científica en el uso de la realidad virtual en la educación a partir de la estructura conceptual, social e intelectual. Gonzalo Lorenzo Lledó

Página 19 de 31 


\section{Figura 10.}

Redes de co-citación entre documentos. Clúster 2. Color Azul.

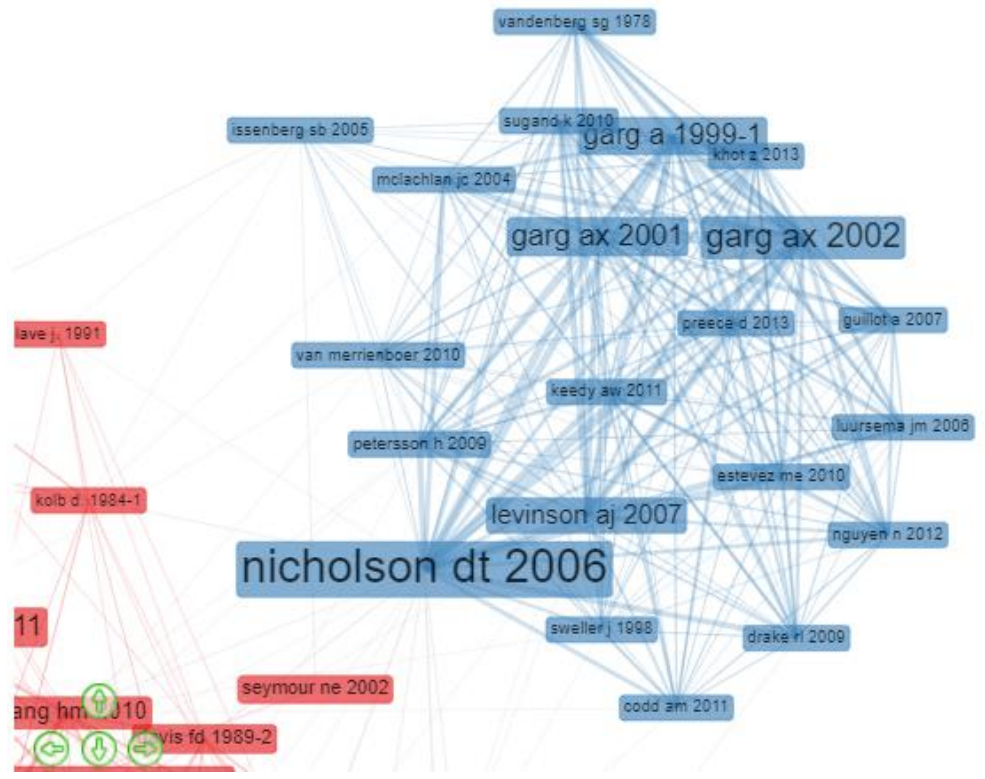

\subsection{1.- Análisis de las palabras clave.}

Con respecto a las palabras claves más utilizadas por las bases de datos (Keyword Plus) y las de los autores (Keyword Authors) existen ciertas diferencias y similitudes. Con respecto a las Keyword Plus una sobresale por encima de todas que es Education. Esta ha sido la más utilizada en diversos periodos en 2007-2008, 2016-2019 y 2011-2012. Asimismo, virtual reality es la que toma la primera posición en los periodos intermedios. Resulta significativo indicar que en el año 2013 se producen un empate entre las dos palabras anteriores y students. En este sentido las cinco palabras más utilizadas configuran un entorno formado por las herramientas virtual reality, las formas de trabajo performance, simulation, los sujetos que son los estudiantes y el campo de estudio que es educación.

En el caso de las Keyword authors, al igual que el caso anterior virtual reality es la palabra más utilizada por los autores prácticamente desde el año 2000. Education adquiere la segunda posición de forma clara excepto en el año 2018 que ocupa la segunda posición con Augmented reality. En el caso de los autores, se habla de dos tipos de herramientas virtual reality, Augmented reality, un campo de trabajo que es education, una forma de trabajo que es simulation. Mientras que el objetivo del campo de estudio es aprendizaje (learning.). 


\subsection{2.- Temas y áreas temáticas.}

Para las Keyword Plus se observa que en el periodo 1994-2007 existen temáticas como educational software, 3-dimensional objects o technology y virtual reality. Por el contrario, en el segundo periodo 2008-2020, la diversidad de temas es más reducida centrada en temas como students, education, medical education y performance. En la figura 11 con respecto al periodo 1994-2007. Dentro de los temas actuales y que ocupan el primer cuadrante destacan en tool (undergraduate, postgraduate, strategy, Outcomes base-education), dentro de student (curriculum, Science, system, training program, computer, radiology, environment), dentro de technology (internal medicine, anatomy, physical examination, deliberate practice).

\section{Figura 11.}

Evolución temática 1994-2007 Keyword Plus

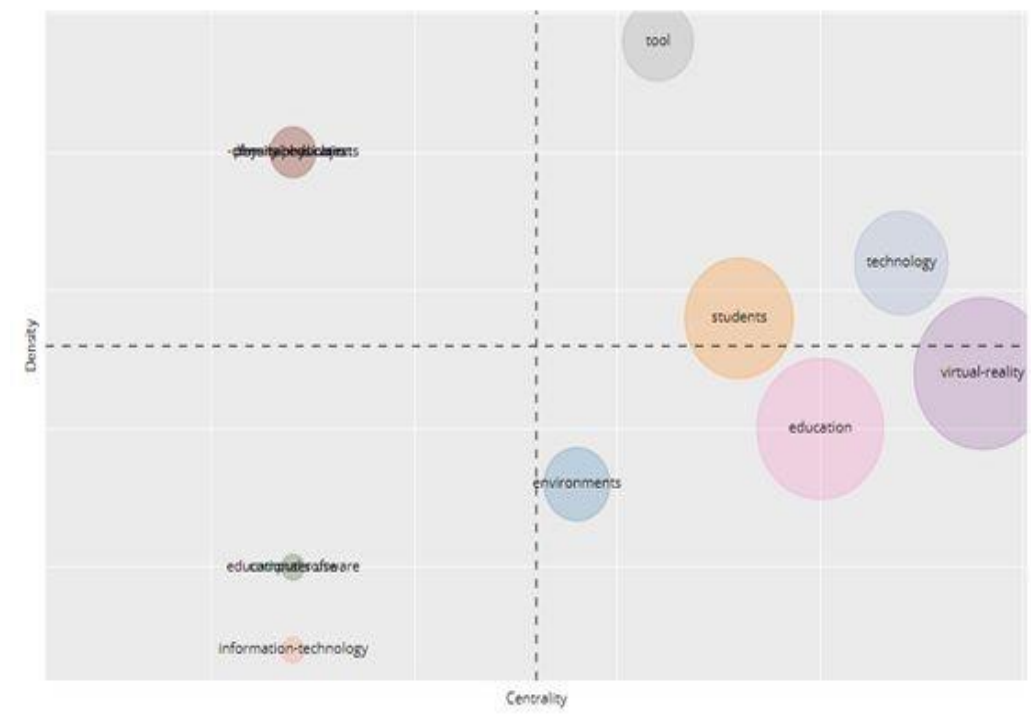

En el periodo 2008-2019 se observa que uno de los temas punteros en este periodo será performance y medical education. Dentro de performance destacan algunos subtemas como simulation, skills, surgery, model, adquistion, validation. Asimismo para medical education destacan tool, anatomy, mental rotation, teaching anatomy, gross anatomy.

En cuanto a las áreas temáticas de las Keyword authors destacan para el primer periodo palabras como computer-mediated-commucation, virtual reality, distance learning, control education, e-learning, undergraduate. En el segundo periodo guarda algunas similitudes con las kewyord Plus como medical education, education y aparecen palabras nuevas como Second Life, learning o virtual. En el primer periodo (figura 12) existe una gran variedad de temáticas, por ejemplo, dentro de undergraduate (hypermedia, 3dmodel, cognitive load, dynamic visualization) para distance learning (remote, data adquistion, computer simulation, mobile robots). Finalmente, para patient simulation destacan temas como Clinical competence, education, medical undergraduate, examination, method

Análisis de la producción científica en el uso de la realidad virtual en la educación a partir de la estructura conceptual, social e intelectual. Gonzalo Lorenzo Lledó 


\section{Figura 12.}

Evolución temática en el primer periodo Keyword authors.

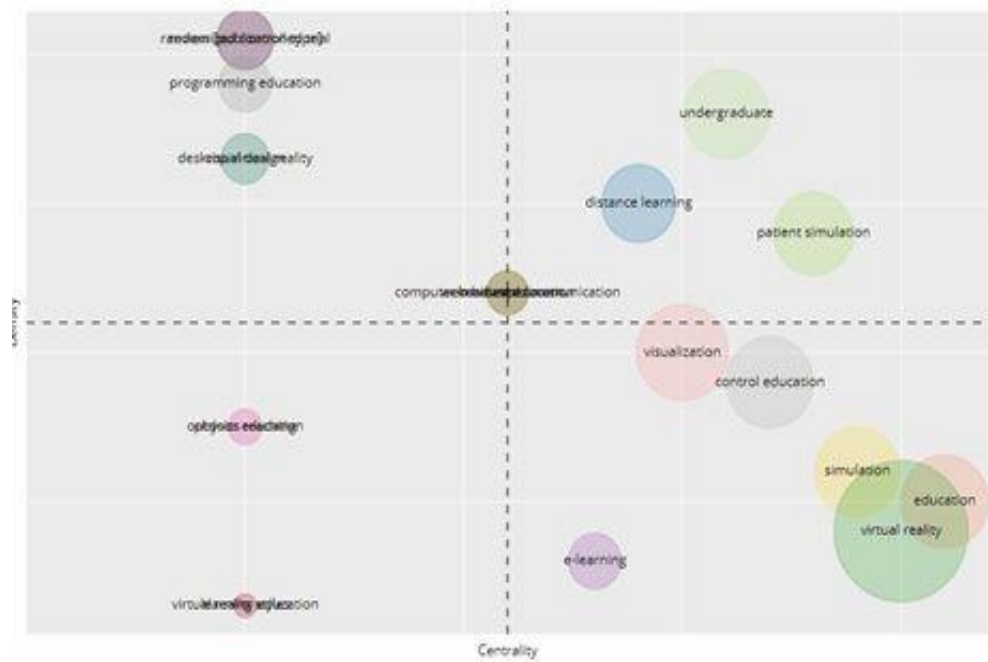

Los temas actuales (figura 13) son medical education que incluye aspectos como teaching, virtual reality technology, visualization, interactivity, e-learning. Para el tema learning sobresalen algunos como dental education, environment, technology enhanced learning.

\section{Figura 13.}

Evolución temática del segundo periodo. Keyword authors.

\section{Discusión}

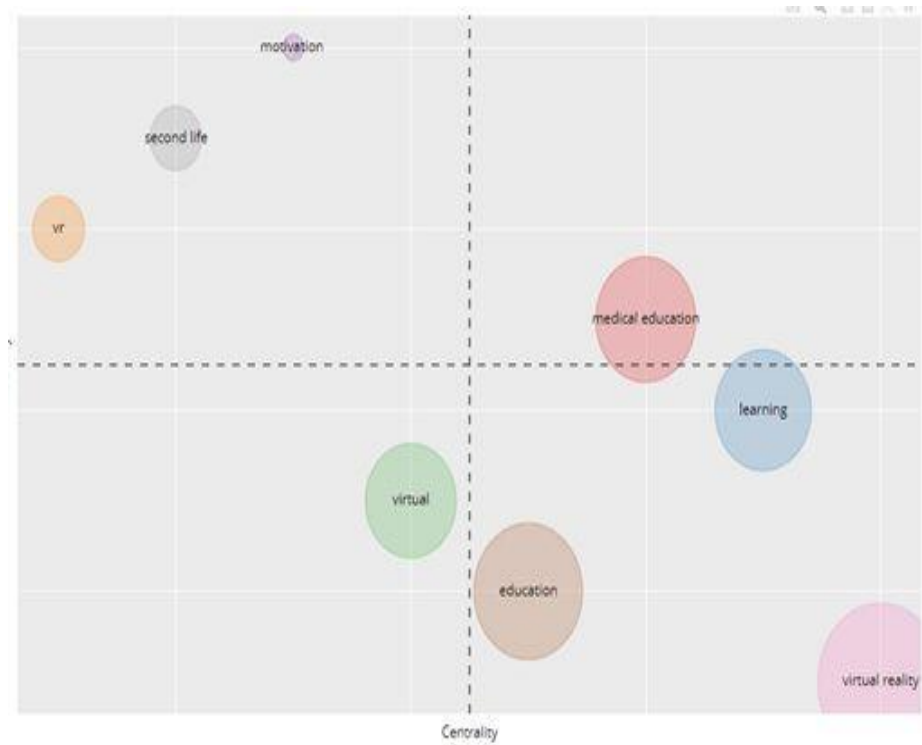

La estructura conceptual de las fuentes está compuesta por dos clústeres de co-citación. Siendo las revistas Computers y Education y Medical Education las que más citas y documentos producen ocupando posiciones centrales de las redes. Esto es consecuencia según Ramos (2012) de que disponen de amplias conexiones sociales dentro del campo.

Análisis de la producción científica en el uso de la realidad virtual en la educación a partir de la estructura conceptual, social e intelectual. Gonzalo Lorenzo Lledó 
En este sentido los valores de closeness de ambos clústeres oscilando entre 20\%-40\%. Dan a entender según Castello-Cogollos et al., (2017) que nos encontramos ante una red descentralizada de revistas agrupadas en torno a la revista que más citas recibe. En línea con lo anterior, la dinámica de las fuentes muestra como Computers y Education en el periodo 2002-2014 es la más productora. Sin embargo, en los últimos años las revistas de medicina han copado las primeras posiciones. Esto es consecuencia de dos factores. El primero según Kavanagh et al., (2017) la producción de dispositivos de RV cada vez más baratos y que hacen posible la difusión de las investigaciones. Mientras que el segundo para González et al., (2018) es que la RV habilita a los profesionales y estudiantes para poder practicar operaciones de cierta complejidad.

En cuanto a la estructura social de los autores, existen dos equipos consolidados que están formados uno de ellos por tres de los autores más productivos, pero no son los más citados. Este resultado va en línea por lo indicado por Hou et al., (2008) que afirman que los autores más productivos son los más activos en las redes. El resto de los clústeres de colaboración lo forman equipos de pequeño tamaño. Con relación a la evolución temporal de la producción de los autores, se observa que en el periodo de 2014-2019 es cuando los 4 autores más productivos generan sus documentos y acumulan más citas. La situación científica de este periodo puede ser debida según Newman (2018) a los recientes avances de Internet como la creación de la red 5G. De esta forma se ha podido trabajar con la RV a velocidades y capacidades de tráfico exponenciales mayores y su expansión a diversos ámbitos como la educación. Esta es la culminación de un proceso de aplicación de la RV en el mundo educativo que empezó hace 20 años (McGovern et al., 2020).

Siguiendo con el estudio, en cuanto la estructura intelectual está compuesta por tres clústeres de co-citación de autores. Al contrario de los indicado por Hou et al., (2008), en este estudio los valores más elevados de btw en las redes de co-citación no corresponden con los autores más productivos. Lo que si se cumple según Brandes et al., (2016) es que los valores más elevados de btw ocupan posiciones centrales en la red, como puedes ser el caso de Mayer. Asimismo, se observa como los equipos de autores más consolidados son los que más producen, pero no los más citados. Siendo consecuencia según Garfield et al., (1978) a que los autores que más citas reciben plasman en sus documentos las ideas más críticas dentro del campo de estudio. Finalmente, en las redes de colaboración entre países, se puede observar como Estados Unidos e Inglaterra son los que presentan los valores de btw más elevados, esto según Hou et al., (2008) los lleva a ocupar posiciones centrales en la red. Esta hace que el resto de los países tiendan a buscar colaboraciones con estos países (Wagner y Leydesdorff, 2005) ya que tienen gran influencia en el campo de trabajo. Como resultado, los clústeres más numerosos son los que cuentan con estos países.

Con relación a la estructura intelectual de los documentos publicados, el autor cuyo documento más citas recibe no se encuentra ni siquiera entre los 50 autores más productivos, pero sí que forma parte de uno de los clústeres más importantes. Esto puede ser debido según Ascaso (2010) a que las revisiones científicas siempre van a recibir más citaciones que los trabajos originales. Esto mismo ocurre con el trabajo de Merchant et al., (2014), Wu et al., (2013). Resulta significativo que entre los documentos más citados no se encuentra ninguno de los autores líderes en el campo y que tiene el índice h más elevado que son Wilson y Darzi. Esto puede ser debido a que los documentos que reciben

Análisis de la producción científica en el uso de la realidad virtual en la educación a partir de la estructura conceptual, social e intelectual. Gonzalo Lorenzo Lledó

Página 23 de $\mathbf{3 1}$ 
más citas presentan investigaciones que son innovadoras y provocan mejoras en el campo (Garfield et al., 1978). Asimismo, existe un documento, el de Nicholson et al., (2010) que reciben una gran cantidad de citas locales. Esas citas según Aria y Curruculo (2017) hacen referencia a la colección en la que se encuentran. Esto nos indica que nos encontramos ante un artículo de referencia en la revista Medical Education. La estructura de co-citación de los documentos está organizada en dos clústeres, siendo en el primer clúster el de Merchant et al., (2014) y el segundo clúster está encabezado por Nicholson et al., (2010). El clúster de Merchant et al., (2014) trabaja temas relacionados con la aplicación de la realidad virtual y aumentada en procesos educativos. Mientras que el clúster de Nicholson et al., (2010), se centra más en proceso de aprendizaje en entornos de medicina. Esta separación en función de los documentos sigue la línea planteada por Small (2004) donde los clústeres se organizan en torno a los documentos más citados.

En referencia a los temas y áreas temáticas que trabajan los documentos se han dividido en dos periodos: el primero de ellos abarca de 1994-2007 mientras que el segundo periodo comprende de 2008-2019. El periodo de 1994-2007 tiene sus temas centrados en el aprendizaje mediante el uso de las tecnologías de forma general tanto para las keyword Plus como para las Keyword authors. Mientras que en el segundo periodo existe un uso más concreto de la realidad virtual tanto para el apartado de la medicina como para los entornos educativos. Como indica Zhang et al., (2016) esta coincidencia en los temas puede venir influenciada por el hecho de que en la mayoría de los casos las palabras que se utiliza del título y Abstract (Keyword Plus), suelen coincidir con las que utilizan los autores (Keyword authors). Además, como indica Autor et al., (2019) este periodo coincide con la ausencia de aplicaciones de RV adaptado al alumnado. Preponderando un enfoque centrado en su utilización como herramienta de videojuego.

El segundo periodo de 2008-2019 se caracteriza por una mayor difusión y convencimiento por parte de los investigadores que la RV puede ser aplicada de forma útil en los procesos de aprendizaje por eso aparecen temáticas como el Curriculum, el estudiante, el aprendizaje. Esto está bastante recogido en las temáticas trabajadas en las Keyword authors. Mientras que las (Keyword Plus) se han centrado más como indica Zhang et al., (2016) en metodologías de aprendizaje. Como pueda ser la investigación de Autor et al., (2016). Esta división temática puede estar justificada por Kavanagh et al., (2017) que afirma que gracias a la creación de dispositivos baratos y portables de RV, su difusión ha sido mayor en diversos campos. Es significativo que este segundo periodo disponga de un elevado número de revisiones que nos indica un campo que está en continuo crecimiento.

\section{Conclusiones}

Con el presente trabajo se ha podido constatar que la producción científica sobre el uso de la realidad virtual en la educación durante el periodo de 1996-2019, está en continuo crecimiento y son cada vez más los ámbitos de conocimiento que la utiliza como una herramienta para los procesos de aprendizaje y la enseñanza. Tomando como referencia el estudio realizado y en función de los resultados obtenidos, se presentan las siguientes conclusiones que dan respuesta a las preguntas planteadas.

Análisis de la producción científica en el uso de la realidad virtual en la educación a partir de la estructura conceptual, social e intelectual. Gonzalo Lorenzo Lledó

Página 24 de 31 
- En la estructura conceptual de las fuentes, se definen dos líneas de trabajo la primera Tecnología y Educación alrededor de la revista Computers y Education. Mientras que la segunda se desarrolla sobre Medicina y Educación en torno a la revista Medical Education.

- En referencia a la estructura intelectual y social de los autores, existen dos clústeres de colaboración formados por los autores más productivos. Por el contrario, estos no disponen de ningún clúster de co-citación del que formen parte.

- En lo referido a la organización social de los países, USA y UK son los países dominantes en los clústeres y el resto se agrupan a su alrededor para posibles colaboraciones.

- La estructura intelectual de los documentos está formada por dos clústeres que están organizados en función del documento de Merchant et al., (2014) y el de Nicholson et al., (2006).

- La estructura conceptual de los documentos muestra que durante el periodo 19942007 la RV estaba poco incluida en los procesos de aprendizaje. Mientras que durante el periodo 2008-2019, se ha producido un mayor incremento de uso en diversos campos de conocimiento.

De los hallazgos obtenidos se puede concluir que el uso de la realidad virtual en el contexto educativo está focalizado de manera incipiente en la educación y más consolidada en el ámbito de la enseñanza de la medicina. De igual manera, se ha podido obtener una imagen sobre que documentos han sido los pioneros de nuevas ideas e intervenciones. En función de los cuales se han llevado a cabo nuevos estudios. Las conclusiones obtenidas pueden ser el punto de partida para la elaboración de un protocolo de investigación sobre qué pasos seguir a la hora de comenzar la implementación de una intervención con realidad virtual en educación. Por ello, se podría establecer en primer lugar la línea de trabajo a través de la información sobre la estructura conceptual de las fuentes. Tras fijar la línea de trabajo, con la estructura intelectual y social de los autores, en segundo lugar, conseguir información sobre aquellos autores y equipos que son pioneros en el campo y por tanto tendrán las investigaciones más innovadoras. En tercer lugar, a partir de la estructura intelectual de los documentos de los autores más importantes analizar aquellas publicaciones que disponen del conocimiento fundamental en el campo. Para finalizar, la estructura conceptual de los documentos hará posible determinar el periodo de tiempo en el que existe más probabilidad de que encontremos aquellos documentos más actuales. Como líneas de investigación futuras, se plantea la posibilidad de crear un software que permita establecer una representación más detallada del campo de estudio, como por ejemplo determinar cuantitativamente las características de los instrumentos utilizados o la edad media de los participantes en todos los estudios de investigación. De este modo, sería posible obtener una imagen general de los tipos de actividades más desarrolladas, las áreas trabajadas o los objetivos planteados.

Presentación del artículo: 26 de noviembre de 2021 Fecha de aprobación: 30 de diciembre de 2022 Fecha de publicación: 30 de enero de 2022

Análisis de la producción científica en el uso de la realidad virtual en la educación a partir de la estructura conceptual, social e intelectual. Gonzalo Lorenzo Lledó

Página 25 de 31 
Lorenzo, G. (2022). Análisis de la producción científica en el uso de la realidad virtual en la educación a partir de la estructura conceptual, social e intelectual. RED. Revista Educación a Distancia, 22(69). http://dx.doi.org/10.6018/red.502601

\section{Financiación}

Esta investigación no ha recibido ninguna subvención específica de los organismos de financiación en los sectores públicos, comerciales o sin fines de lucro.

\section{6.-Referencias}

Abi-Rafeh, J., Zammit, D., Jaberi, M., Al-Halabi, B., y Thibaudeau, S. (2019). Nonbiological Microsurgery Simulators in Plastic Surgery Training: A Systematic Review. Plastic and Reconstructive Surgery, 144(3), 496-507. https://doi.org/10.1097/PRS.0000000000005990.

Acedo, F. J., Barroso, C., Casanueva, C., y Galan, J. L. (2006). Co-authorship in management and organizational studies: An empirical and network analysis. Journal of Management Studies, 43 (5), 957-983. https://doi.org/10.1111/j.14676486.2006.00625.x

Aria, M., y Cuccurullo, C. (2017). Bibliometrix: An R-tool for comprehensive science mapping analysis. Journal of Informetrics, 11(4), 959-975. https://doi.org/10.1016/j.joi.2017.08.007

Aria, M., Misuraca, M., y Spano, M. (2020). Mapping the Evolution of Social Research and Data Science on 30 Years of Social Indicators Research. Social indicators research, 149 (1), 803-831. https:// doi.org/ 10.1007/s11205-020-02281-3

Ascaso, F. (2010). H-index in the evaluation of individual scientific output. Archivos de la Sociedad española de oftalmologia, 85 (9), 310. https://doi.org/10.1016/S21735794(10)70050-5

Brandes, U., Borgatti, S., y Freeman, L. (2016). Maintaining the duality of closeness and betweenness centrality. Social Networks, $44 \quad$ (1), 153159.https://doi.org/10.1016/j.socnet.2015.08.003

Biocca, F. (1992). Communication within virtual reality: Creating a space for research. Journal of Communication, 42 (4), 5-22. https://doi.org/10.1111/j.14602466.1992.tb00810.x

Burdea, G., y Coiffet, P. (1994). Virtual reality technology (1st Ed.). London: WileyInterscience.

Bower, M., DeWitt, D., and Lai, J. W. (2020). Reasons Associated with Preservice Teachers' Intention to Use Immersive Virtual Reality in Education. Br. J. Educ. Technol. 51 (6), 2214-2232. doi:10.1111/bjet.13009

Callon, M., Courtial, J., Turner, W., y Bauin, S. (1983). From translations to problematic networks: An introduction to co-word analysis. Social Science Information, 22, 191235. https://doi.org/10.1177/053901883022002003

Chávez B., y Bayona S. (2018). Virtual Reality in the Learning Process. In: Rocha Á., Adeli H., Reis L., Costanzo S. (eds) Trends and Advances in Information Systems and Technologies. WorldCIST'18. Advances in Intelligent Systems and Computing (pp.1345-1356). Springer: Cham. https://doi.org/10.1007/978-3-319-77712-2_129

Análisis de la producción científica en el uso de la realidad virtual en la educación a partir de la estructura conceptual, social e intelectual. Gonzalo Lorenzo Lledó 
Costas, R. y Bordons, M. (2007). Una visión crítica del índice h: algunas consideraciones derivadas de su aplicación práctica. El profesional de la información, 16(5), 427-432. DOI: 10.3145/epi.2007.sep.0

Cuccurullo, M., Aria, M., y Sarto, M. (2016). Foundations and trends in performance management. A twenty-five-year bibliometric analysis in business and public administration domains. Scientometrics, 108 (1), 595-611. https://doi.org/ $10.1007 / \mathrm{s} 11192-016-1948-8$

Cunningham, D., y Duffy, T. (1996). Constructivism: Implications for the design and delivery of instruction. In Handbook of research for educational communications and technology (pp. 170-198).

De Moya-Anegón, F., Vargas-Quesada, B., Chinchilla-Rodríguez, Z., Herrero-Solana, V., y Corera-Álvarez, E. (2006). Análisis de la cobertura de la base de datos Scopus. El Profesional de la información, 15 (2), 142-144.

Diem, A., y Wolter, S. (2013). The use of bibliometrics to measure research performance in Education Science. Research in Higher Education, 54 (1), 86-113. https://doi.org/10.1007/s11162-012-9264-5

Donthu, N., Kumar, S., Mukherjee, D., Pandey, N., y Lin, W. (2021). How to conduct a bibliometric analysis: An overview and guidelines. Journal of Business Research, 133(1), 285-296. https://doi.org/10.1016/j.jbusres.2021.04.070

Ezugwu, A.E., Shukla, A.K., Agbaje, M., Oyelade, O., Jose-García, A., y Agushaka, J. (2021). Automatic clustering algorithms: a systematic review and bibliometric analysis of relevant literature. Neural Computing \& Application, 33(1), 62476306.https://doi.org/10.1007/s00521-020-05395-4

Fernández-Cruz, F.J., y Fernández -Díaz, M.J. (2018). El proceso de integración y uso pedagógico de las TIC en los centros educativos madrileños. Educación XX1, 21 (2), 395-416. https://doi.org/10.5944/educXX1.17907.

Garfield, E., Malin, M., y Small, H. (1978). Citation data as science indicators. Y. Elkana, J. Lederberg, A. Thackray, R. Merton, H. Zuckerman (eds). Toward a metric science: The advent of science indicators (pp. 580-608). Wiley: New York.

Glänzel, W. (2002). Coauthorship patterns and trends in the sciences (1980-1998): A bibliometric study with implications for database indexing and search strategies. Library Trends, 50 (3), 461-473.

González, S., Juanes, J., García-Peñalvo, F., Gonçalves, J., Sánchez, J., y Ruisoto, P. (2018). Virtual Reality as an Educational and Training Tool for Medicine. Journal of Medical Systems, 42 (50), 1-5. https://doi.org/10.1007/s10916-018-0900-2

Hanson, J., Andersen, P., y Dunn, P. (2019). Effectiveness of three-dimensional visualisation on undergraduate nursing and midwifery students' knowledge and achievement in pharmacology: A mixed methods study. Nurse Education Today, 81, 19-25. https://doi.org/10.1016/j.nedt.2019.06.008

Hamilton, D., McKechnie, J., Edgerton, E., y Wilson, C. (2021). Immersive virtual reality as a pedagogical tool in education: a systematic literature review of quantitative learning outcomes and experimental design. J. Comput. Educ. 8 (1), 1-32 https://doi.org/10.1007/s40692-020-

Huttar, C., y Brintzenhofeszoc, K. (2019). Virtual Reality and Computer Simulation in Social Work Education: A Systematic Review. Journal of Social Work Education, 111. https://doi.org/10.1080/10437797.2019.1648221

Análisis de la producción científica en el uso de la realidad virtual en la educación a partir de la estructura conceptual, social e intelectual. Gonzalo Lorenzo Lledó

Página 27 de 31 
Hutton, B., Catalá-López, F., y Moher, D. (2016). The PRISMA statement extension for systematic reviews incorporating network meta-analysis: PRISMA-NMA. Medicina Clínica, 147(6), 262-266. DOI: 10.1016/j.medcli.2016.02.025

Holden, G., Rosenberg, G., y Barker, K. (2005). Bibliometrics in social work. Binghamton, NY: Haworth Press.

Hou, H., Kretschmer, H., y Liu, Z. (2008). The structure of scientific collaboration networks in Scientometrics. Scientometrics, $75 \quad$ (2), 189202. https://doi.org/10.1007/s11192-007-1771-3

Hwang, G.-J., Jong, M. S. Y., y Shang, J. (2018). Call for papers. Interactive Learning Environments, 26(4), 566

Issenberg, S., McGaghie, W., Petrusa, E., Lee, D., Scalese, R. (2005). Features and uses of high-fidelity medical simulations that lead to effective learning: A BEME systematic review. Medical Teacher, 27(1), 10-28.

Jensen, L., Konradsen, F. (2018). A review of the use of virtual reality head-mounted displays in education and training. Educ Inf Technol 23, 1515-1529 https://doi.org/10.1007/s10639-017-9676-0

Johnson, A., Roussos, M., Leigh, J., Vasilakis, C., y Barnes, C.; Moher, T. (1998). The NICE project: learning together in a virtual world. In Proceedings of the IEEE virtual reality Annual International Symposium, (pp.176-183). Atlanta: Institute of Electrical and Electronic Engineering (IEEE)

Kamada, T., y Kawai, S. (1989). An algorithm for drawing general undirected graphs. Information Processing Letters, 31 (1), 7-15.https://doi.org/10.1016/00200190(89)90102-6

Kavanagh, S., Luxton-Reilly, A., Wuensche, B., y Plimmer, B. (2017). A systematic review of Virtual Reality in education. Themes in Science and Technology Education, $10(2), 85-119$.

Kim, M., y Hall, C. (2019). A hedonic motivation model in virtual reality tourism: Comparing visitors and non-visitors. International Journal of Information Management, 46, 236-249. https://doi.org/10.1016/j.ijinfomgt.2018.11.016

Lee, E., y Wong, K. (2014). Learning with desktop virtual reality: Low spatial ability learners are more positively affected. Computers y Education, 79 (1), 49-58. https://doi.org/10.1016/j.compedu.2014.07.010

Liu, D., Bhagat, K., Gao Y., Chang TW., y Huang R. (2017) The Potentials and Trends of Virtual Reality in Education. In: Liu D., Dede C., Huang R., Richards J. (eds) Virtual, Augmented, and Mixed Realities in Education. Smart Computing and Intelligence (pp.105-130). Springer: Singapore. https://doi.org/10.1007/978-981-105490-7_7

Lorenzo, G., Lledó, A., Pomares, J., y Roig, R. (2016). Design and application of an immersive virtual reality system to enhance emotional skills for children with autism spectrum disorders. Computers y Education, 98 (1), 192-205. https://doi.org/10.1016/j.compedu.2016.03.018

Lorenzo, G., Lledó, A., Arráez-Vera, G., y Lorenzo-Lledó, A. (2019). The application of immersive virtual reality for students with ASD: A review between 1990-2017. Education and Information Technologies, 24 (1), 127-151. https://doi.org/10.1007/s10639-018-9766-7

Lorenzo, G., Lorenzo-Lledó, A., Lledó, A., y Pérez-Vázquez, E. (2020). Application of virtual reality in people with ASD from 1996 to 2019. Journal of Enabling Technologies, 14 (2), 99-114. https://doi.org/10.1108/JET-01-2020-0005

Análisis de la producción científica en el uso de la realidad virtual en la educación a partir de la estructura conceptual, social e intelectual. Gonzalo Lorenzo Lledó 
Manterola, C., Astudillo, P., Arias, E., y Claros, N. (2013). Revisiones sistemáticas de la literatura. Qué se debe saber acerca de ellas. Cirugía Española, 91(3), 149-155. DOI: 10.1016/j.ciresp.2011.07.009

Marín, V., y Muñoz, V. P. (2018). Trabajar el cuerpo humano con realidad aumentada en educación infantil. Revista Tecnología, Ciencia y Educación, 9, 148-158. https://doi.org/10.51302/tce.2018.177

Martínez, M.A., Cobo, M., Herrera, M., y Herrera-Viedma, E. (2015). Analyzing the Scientific Evolution of Social Work Using Science Mapping. Research on Social Work using Science mapping. Research on Social Work Practice, 25 (2), 257-277. https://doi.org/10.1177/1049731514522101

McCain, K. W. (1990). Mapping authors in intellectual space: A technical overview. Journal of the American Society for Information Science and Technology, 41 (6), 43343. https://doi.org/10.1002/(SICI)1097-4571(199009)41:6<433::AIDASI11>3.0.CO;2-Q

Mc Donald, D., Crabtree, J. R., Wiesinger, G., Dax, T., Stamou, N., Fleury, P., GutierrezLazpita, J., y Gibon, A. (2000). Agricultural abandonment in mountain areas of Europe: environmental consequences and policy response. Journal of Environmental Manage, 59 (1), 47-69. https:// doi.org/10.1006/jema.1999.0335.

McGovern, E., Moreira, G., y Luna-Nevarez, C. (2020). An application of virtual reality in education: Can this technology enhance the quality of students' learning experience? Journal of Education for Business, 95 (7), 490496.https://doi.org/10.1080/08832323.2019.1703096

Merchant, Z., Goetz, E., Cifuentes, L., Keeney, W., y Davis, T. (2014). Effectiveness of virtual reality-based instruction on students' learning outcomes in K-12 and higher education: A meta-analysis. Computers \& Education, 70 (1), 29-40. https://doi.org/10.1016/j.compedu.2013.07.033

Moher, D., Shamseer, L., Clarke, M., Ghersi, D., Liberatl, A., Petticrew, M., Shekelle, P. y Stewart, L. (2015). Preferred reporting items for systematic review and meta-analysis protocols (PRISMA-P) 2015 statement. Systematic Reviews, 4(1), 1 - 9. doi: 10.1186/2046-4053-4-1

Newbutt, N., Sung, C., Kuo, H., Leahy, M., Lin, C., y Tong, B. (2016). Brief Report: A Pilot Study of the Use of a Virtual Reality Headset in Autism Populations. Journal of Autism and Developmental Disorders, 46 (1), 3166-3176. https://doi.org/10.1007/s10803-016-2830-5

Newman, D. (2018). 4 Reasons 5 G Is Critical for Mass Adoption of AR and VR. Fortune Magazine, Mar 27th, 2018. Recuperado de https://www.forbes.com/sites/daniel newman/2018/03/27/4-reasons-5g-is-critical-for-mass-adoptionof-ar-andvr/\#501f9ac01878

Nicholson, D., Chalk, C., Funnell, R. y Daniel, S. (2006). Can virtual reality improve anatomy education? A randomised controlled study of a computer-generated threedimensional anatomical ear model. Medical Education, 40 (11), 1081-1087.

Noyons, E., Moed, H., y Luwel, M. (1999). Combining mapping and citation analysis for evaluative bibliometric purposes: A bibliometric study. Journal of the American Society for Information Science, 50, 115-131.https://doi.org/10.1002/(SICI)10974571(1999)50:2<115::AID-ASI3>3.0.CO;2-J

Page, M., McKenzie, J., Bossuyt, P., Boutron, I., Hoffmann, T., Mulrow, C., Shamseer, L., Tetzalaff, J., Akl, E., Brenan, S., Chou, R., Glanville, J., Grimshaw, J., Hróbjartsson, A., Lalu, M., Li, T., Loder, E., Mayo-Wilson, E., McDonald, S.,

Análisis de la producción científica en el uso de la realidad virtual en la educación a partir de la estructura conceptual, social e intelectual. Gonzalo Lorenzo Lledó

Página 29 de 31 
McGuinness, L., Stewart, L., Thomas, J., Tricco, A., Welch, V., y Moher, D.(2021). Declaración Prisma 2020: una guía actualizada para la publicación de revisiones sistemáticas. Revista Española de Cardiología, 74(9), 790-799.

Parsons, S., y Cobb, S. (2011). State-of-the-art of virtual reality technologies for children on the autism spectrum. European Journal of Special Needs Education, 26 (3), 355366. https://doi.org/10.1080/08856257.2011.593831

Pérez, R., Mercado, P., Martínez, M., Mena, E., y Partida, J.A. (2018). La Sociedad del conocimiento y la Sociedad de la Información como la piedra angular en la innovación tecnológica educativa. Revista Iberoamericana para la Investigación y el desarrollo educativo, 8 (16), 1-24.

Pons, P., y Latapi, M. (2006). Computing communities in large networks using random walks. Journal of Graph Algorithms and Applications, 10 (2), 191-218. https: //doi.org /10.7155/jgaa.00124

Pradhan, T., y Pal, S. (2020). A hybrid personalized scholarly venue recommender system integrating social network analysis and contextual similarity. Future Generation Computer Systems, 110 (1), 1139-1166.https://doi.org/10.1016/j.future.2019.11.017

Radianti, J., Majchrzak, T., Fromm, J., y Wohlegenannt, I. (2020). A systematic review of immersive virtual reality applications for higher education: Design elements, lessons learned, and research agenda. Computers \& Education, 147 (1), 1-29. https://doi.org/10.1016/j.compedu.2019.103778

Rahman, M. (2017). Basic Graph Terminologies. In M. Rahman (Eds), Basic Graph Theory (pp.11-29). Switzerland. Springer International Publishing

Ramos, C. (2012). Estructuras de comunicación en el campo de la ciencia social en Chile: un análisis de redes. Redes: Revista Hispana para el Análisis de Redes Sociales, 1 (23), 7-42. https://doi.org/10.5565/rev/redes.438

Rodríguez-García, A., Trujillo, J.M., y Sánchez, J. (2019). Impacto de la productividad científica sobre competencia digital de los futuros docentes: aproximación bibliométrica en Scopus y Web of Science. Revista Complutense de Educación, 30 (2), 626-646. https://doi.org/10.5209/RCED.58862

Rodríguez-Soler, R., Uribe-Toril, J., y De-Pablo Valenciano, J. (2020). Worldwide trends in the scientific production on rural depopulation, a bibliometric analysis using Bibliometrix R-tool. Land Use Policy, $97 \quad$ (1), 1-20. https://doi.org/10.1016/j.landusepol.2020.104787

Repiso, R., y Torres-Salinas, D. (2016). Características e implicaciones de la base de datos Emerging Sources Citation Index (Thomson Reuters): las revistas en estado transitorio. Anuario Thinkepi, 10 (1), 234-236. https: //doi.org/10.3145/thinkepi.2016.46

Sanguri, K., Bhuyan, A., y Patra, S. (2020). A semantic similarity adjusted document cocitation analysis: a case of tourism supply chain. Scientometrics 125 (1), 233-269 (2020). https://doi.org/10.1007/s11192-020-03608-0

Small, H. (1973). Co-citation in the Scientific literature: A new measure of the relationship between two documents. Journal of the American Society for Information Science, 24 (4), 28-31. https://doi.org/10.1002/asi.4630240406

Small, H. (2004). On the shoulders of Robert Merton: towards a normative theory of $\begin{array}{llll}\text { citation. } & \text { Scientometrics, } & 60 & \text { 71-79. }\end{array}$ https://doi.org/10.1023/B:SCIE.0000027310.68393.bc

Schmidt, M., Schmidt, C., Glaser, N., Beck, D., Lim, M., y Palmer, H. (2021). Evaluation of a spherical video-based virtual learning intervention to teach adaptive skills for

Análisis de la producción científica en el uso de la realidad virtual en la educación a partir de la estructura conceptual, social e intelectual. Gonzalo Lorenzo Lledó

Página 30 de 31 
adults with autism: a preliminary report. Interactive Learning Environments, 29(3), 345-364. DOI: 10.1080/10494820.2019.1579236

Sporns, O. (2018). Graph Theory methods: applications in brain networks. Dialogues Clin Neurosci. 20 (2), 111-121.https://doi.org/ 10.31887/DCNS.2018.20.2/ospornsL

Sural, I. (2018). Augmented Reality Experience: Initial Perceptions of Higher Education Students. International Journal of Instruction, $11 \quad$ (4), 565-576. https://doi.org/10.12973/iji.2018.11435a

Urrutia, G., y Bonfill, X. (2010). Declaración PRISMA: una propuesta para mejorar la publicación de revisiones sistemáticas y metaanálisis. Medicina Clínica, 135(11), 507511. DOI: 10.1016/j.medcli.2010.01.015

Viñals, A., y Cuenca, J. (2016). El rol del docente en la era digital. Revista Interuniversitaria de Formación del Profesorado, 30 (2), 103-114. https://doi.org/10.47553/rifop.v30i2

White, H. D., y Griffith, B. C. (1981). Author cocitation: A literature measure of intellectual structure. Journal of the American Society for Information Science, 32 (3), 163-171. https://doi.org/10.1002/asi.4630320302

Wu, H., Wen, S., Chang, H., y Liang, J. (2013). Current status, opportunities and challenges of augmented reality in education. Computers \& Education, 62 (1), 41-49. https://doi.org/10.1016/j.compedu.2012.10.024.

Wu, W., Heartless, J., Tesei, A., Gunji, V., Ayer, S., y London, J. (2019). Design Assessment in Virtual and Mixed Reality Environments: Comparison of Novices and Experts. Journal of Construction Engineering and Management, 145 (9), 1-14. https://doi.org/10.1061/(ASCE)CO.1943-7862.0001683

Zhao, D. (2006). Towards all-author co-citation analysis. Information Processing \& Management, 42 (6), 1578-1591. https://doi.org/10.1016/j.ipm.2006.03.022

Zhao, R., Li, D., Wei, M., y Li, X. (2021). The contents and methods of knowledge network from the perspective of bibliometrics. Technology Analysis y Strategic Management. https://doi.org/10.1080/09537325.2021.1894329

Zhang, J., Yu, Q., Zheng, F., Long, C., Lu, Z., y Duang, Z. (2016). Comparing keywords plus of WOS and author keywords: A case study of patient adherence research. Journal of the association for information science and technology, 67 (4), 967-972. https://doi.org/10.1002/asi.23437

Zupic, I., y Cater, T. (2015). Bibliometric methods in Management and Organization. Organizational. Research Methods, 18 (3), 429-472. https://doi.org/10.1177/1094428114562629

Análisis de la producción científica en el uso de la realidad virtual en la educación a partir de la estructura conceptual, social e intelectual. Gonzalo Lorenzo Lledó 